\title{
Multilevel Redundant Discrete Wavelet Transform (ML-RDWT) and optimal Red Deer algorithm (ORDA) centred approach to mitigate the effect of ICI, BER and CIR in a MIMO-OFDM System
}

K Nagarajan ( $\sim$ knagarajan0711@gmail.com )

Anna University Chennai

\section{S Sophia}

Sri Krishna College of Engineering and Technology

\section{Research Article}

Keywords: MIMO-OFDM systems, Multi-level Redundant Discrete Wavelet Transform, Inter Carrier Interference, Inter symbol interference, Bit-error rate, Carrier-to-interference power ratio, Down-Sampling, and Optimal red deer algorithm

Posted Date: March 24th, 2021

DOl: https://doi.org/10.21203/rs.3.rs-235830/v1

License: (c) (1) This work is licensed under a Creative Commons Attribution 4.0 International License. Read Full License

Version of Record: A version of this preprint was published at Wireless Personal Communications on September 12th, 2021. See the published version at https://doi.org/10.1007/s11277-021-09066-y. 


\title{
Multilevel Redundant Discrete Wavelet Transform (ML-RDWT)
}

\section{and optimal Red Deer algorithm (ORDA) centred approach to}

\section{mitigate the effect of ICI, BER and CIR in a MIMO-OFDM System}

\author{
Mr. K. Nagarajan ${ }^{1 *}$, Dr. S. Sophia ${ }^{2}$ \\ ${ }^{1 *}$ Research Scholar, Department of Information and communication Engineering, Anna \\ University Chennai India \\ ${ }^{1 *}$ Assistant Professor, Department of Electronics and communication engineering, Nehru \\ Institute of Engineering and Technology Coimbatore, Tamilnadu, India \\ *Email: knagarajan0711@gmail.com \\ ${ }^{2}$ Professor and Head, Department of Electronics and Communication Engineering, Sri Krishna \\ College of Engineering and Technology, Coimbatore, Tamilnadu, India
}

\begin{abstract}
Nowadays, there is a great demand for ultra-high data rate (UHDR) transmission on most $5^{\text {th }}$ generation wireless networks. In this concern, the multiple-input multiple-output orthogonal frequency division multiplexing (MIMO-OFDM) scheme is used on a large scale to achieve UHDR transmission with reduced inter-symbol interference (ISI) and inter-carrier interference (ICI). Discrete wavelet transform-based OFDM (DWT-OFDM) provides better orthogonality due to presence of orthogonal wavelets, which mitigates the effects caused by ISI and ICI. Also, it has extended bandwidth than the traditional OFDM systems. But a major drawback in this system is that it suffers from down sampling. The down-sampling effect reduces the actual size of the input bit streams. As a result, the system performance is degraded. For solving this problem, a multilevel redundant discrete wavelet transform (ML-RDWT) is used instead of
\end{abstract}


DWT to achieve improved spectral performance. Here, complex down-sampling operation is eliminated. From the simulation outcomes, it is clearly viewed that effects caused by ICI, ISI and BER are mitigated by improving the performance of CIR. The proposed method employs optimal red deer algorithm (ORDA) to locate the optimized weights for the ICI cancellation system. This algorithm enhances the spectral efficiency by achieving high CIR with reduced BER, ISI and ICI. The BER in the proposed MIMO-ML-RDWT-OFDM-ORDA method is 68\%, $76 \%, 38 \%$ and $75 \%$, which is very low when compared to the BER in the existing techniques like MIMO-DWT-OFDM-RDA, MIMO-RNS-OFDM-PNMA, MIMO-OFDM-BMA and MIMO-OFDM-ICIMA. The ISI in the proposed method is $94 \%, 91 \%, 95 \%$ low when compared to the ISI in the existing techniques. The ICI in the proposed work is $71 \%, 57 \%, 73 \%$ and $86 \%$ low when compared to the ICI in the existing techniques. Therefore, the general performance of the proposed MIMO-ML-RDWT-OFDM-ORDA method is improved in an efficient way with less complexity, error rate and processing delay.

Keywords: MIMO-OFDM systems, Multi-level Redundant Discrete Wavelet Transform, Inter Carrier Interference, Inter symbol interference, Bit-error rate, Carrier-to-interference power ratio, Down-Sampling, and Optimal red deer algorithm.

\section{Introduction}

The combination of multiple-input multiple-output (MIMO) and orthogonal frequency division multiplexing (OFDM) serves as powerful tool for many of the broadband wireless access and standards. The MIMO system often suffers from interference between antennas [1]. The channel capacity of the MIMO based system is improved by connecting multiple transmitters and receivers at both ends. Therefore, the MIMO system achieves increased reliability, spectrum efficiency and coverage [2]. The entire channel in an OFDM system is split into numerous 
narrow-band sub channels that transmitted on parallel to preserve the high data rate transmission. The flexibility feature of OFDM improves transmission efficiency, so it is suitable to use in many advanced techniques like adaptive load, transfer, and receiver diversity [3]. OFDM has choosen various high-speed wireless local area network (WLAN) standards like IEEE 802.11a and IEEE $802.11 \mathrm{~g}$ that support data rates up to $54 \mathrm{Mbps}$ [4].

The OFDM system can successfully combat inter-symbol interference (ISI), so it is employed on high data rate communication systems [5]. Another concern is that information theory indicates that increased system capacity may be achieved through deploying multiple antennas for transmitting and receiving ends of systems by suitable space-time signal processing methods [6, 7]. MIMO-OFDM systems consist of multiple front-ends, thus reducing cost, size and power consumption within the suitable limit [8]. The OFDM based MIMO transmission is well suitable to use in broadband wireless technology [9].

The OFDM system may successfully convert a frequency selective (FS) channel [10] into multiple flat frequency sub channels on dissimilar subcarriers to mitigate multipath effects. Currently, it has been selected as fifth generation (5G) waveform for sub-6 GHz [2, 3] with the rating of third generation partnership project (3GPP) [11]. The MIMO-OFDM systems are used everywhere at modern telecommunication systems like Long Term Evolution (LTE) WLAN systems because of their spatial multiplexing property $[12,13]$. Thus, $5 \mathrm{G}$ wireless networks are evolved for reaching ultra-high data rate (UHDR) transmission in an efficient way [14].

The carriers in the OFDM are chosen to be orthogonal for diminishing inter-subcarrier interference and maximize spectral performance. OFDM is a multi-carrier modulation system, which generates orthogonal subcarriers using discrete Fourier transform. 
The MIMO-OFDM system has the following problems:

- ICI is caused by some distortions such as carrier frequency offset and phase noise.

- In addition, the $\mathrm{CP}$ channel is higher than the length can cause ISI

- The BER performance has been degraded as a result of time and frequency synchronization.

- Also, carrier frequency offset (CFO) is a major BER and ICI.

For enhancing the CIR performance, an optimized and suboptimal version of algorithm [15] analyzed in the proposed MIMO-OFDM framework is presented. Though the OFDM systems presented in recent literature use the AWGN channel environment used DWT that undergoes the decimation process when the input signal is broken down into approximate and detail coefficients. The complex down-sampling process diminishes the original size of input bit stream resulting in original data loss on receiver end. This issue is motivated to do this work. In this work, a multilevel discrete wavelet transform is implemented, which is an improved version of RDWT [16] for enhancing the spectral performance of MIMO-OFDM system in an efficient way by mitigating the effects caused with inter-symbol interference (ISI), Inter-carrier Interference (ICI) and Bit Error Rate (BER) with increased carrier-to-interference power ratio (CIR).

The growing demand for fifth-generation wireless networks with diminished ISI and ICI for efficient data transmission is possible with the use of MIMO-OFDM. The MIMO-OFDM based scheme is well suited to achieve UHDR transmission.

The main contribution of this work is summarized as follows:

In this work, to propose a Multilevel Redundant Discrete Wavelet Transform (ML-RDWT) in a MIMO-OFDM framework by fading channel environment and power delay profile. 
- The main goal is to improve the spectral performance by mitigating the effects caused by ICI, ISI and BER efficiency in fading conditions and power delay profile that is not focused on previous investigation work done on this area.

- To diminish the Inter Carrier Interference (ICI), Bit Error Rate (BER) and enhancing the CIR is proposed utilizing the Optimal Red Deer Algorithm (ORDA) for time varying fading channel.

- The proposed system operates in two easy steps. On transmitter side, a data symbol is modulated by a weighting coefficients group into a group of adjacent subcarriers. [17, $18]$.

- In residual ICI reduction is obtained on receiver signal to a considerable level as interference is cancelled. The weighting coefficients are intended in these ways that the result of carrier frequency offset on subcarriers may be diminished.

- At the receiver side, the residual ICI contained on receiver signals is more diminished by linearly combining the received signals on the subcarriers along with the weighting coefficients.

- The CIR may be improved based on group size of a channel through constant frequency offset (FO).

- The use of optimal Red Deer algorithm (ORDA) helps to find the optimized weights for mitigating the effects caused by ICI, ISI, BER and CIR. Therefore, the overall performance of proposed MIMO-ML-RDWT-OFDM system may be improved in an efficient way with high spectral efficiency.

The paper is mentioned as beneath: First section deals with introduction about MIMOOFDM systems. Second section deals with some of the important related works carried out to 
mitigate the effects caused by ICI, ISI and BER in a MIMO-OFDM System. In third section, a detailed description about the proposed Multilevel Redundant Discrete Wavelet Transform (MLRDWT) in a MIMO-OFDM framework is presented with optimal red deer (ORDA) algorithm to optimize the weight parameters for achieving enhanced spectral efficiency by improving CIR performance in a MIMO-OFDM System. Section four deals with the simulation results obtained from the proposed method and they are compared with existing system to show the performance of proposed MIMO-ML-RDWT-OFDM-ORDA technique. Finally, section five concludes the paper with some references.

\section{Related Work: A Brief Review}

Some of the most recent research works about MIMO-OFDM system were reviewed here in this section.

Lu (2017) et.alin [19] has focused on the high speed railway (HSR) downlinks with distributed transmit antennas and evolve two related ICI reduction systems for additive white Gaussian noise (AWGN) and Rician channels. Through the information of the relative locations and speed among equivalent antenna pairs, they illustrate ICI matrices on AWGN and Rician channels may be mathematically computed and unity. With these outcomes, they introduce two equivalent minimum-complexity ICI reduction systems for preventing matrix inversion and adapt to rapid time-varying nature. The simulation outcomes demonstrate their ICI mitigation system may accomplish an amount of equal service obtained in case without ICI while the speed is approximately $300 \mathrm{~km} / \mathrm{h}$.

Hao (2016) et.al in [20] has introduced a low complexity ICI mitigation system for MIMOOFDM systems in assumption of linear channels that vary over time. This diminishes the ICI compensation complexity and needs channel rating depend on time-varying linear channel 
model, and does not require transmission overhead. Synchronous time domain OFDM was naturally suited to introduce ICI mitigation mechanism since their receiver time may simply assess linear channels. The simulation with QPSK and 16 QAM modulation shows that efficiency of an introduced system, compared with no ICI mitigation case.

Nakamura (2018) et.al in [21] suggested an MIMO-OFDM system with dual polarization to use in Japanese digital terrestrial television broadcasting systems. The interference between ICI that was created due to Doppler dispersion on mobile reception of MIMO-OFDM systems is considered as a main issue. The MIMO-ICI canceller depends on zero forcing (ZF) and it diminishes complex computations like matrix operations. The ZF-based complexity reduction MIMO-ICI canceller may enhance the ICI influence through less complexity. Furthermore, MIMO-ICI cancellers depends on minimum mean square error (MMSE) are suggested. As a result of computer simulations, MIMO-ICI cancellation messages with ZF and MMSE-based repetitive detection may enhance less complex reception properties.

Paek (2019) et.al in [22] has introduced a performance improvement system with coordinated multi-point (CoMP) through spatial phase coding (SPC) depends on MIMO - OFDM on heterogeneous network system (HetNet). At conventional system, the mobile terminal (MT) efficiency degrades based on inter-carrier interference (ICI). While the MT was placed at the edge of cell, the efficiency and quality of service (QoS) of MT was attenuated based on interference caused with signal transmitted as adjacent base station (BS) or signal transmitted with other MTs. For maximizing the MT reliability, an introduced system utilizes a pre-coding system and CoMP on HetNet. The simulation outcomes demonstrate that introduced system has enhanced BER efficiency and greater performance to conventional system. 
Pham (2016) et.al in [23] have introduced a repetitive structure of channel estimation and data detection for MIMO-OFDM systems through an inappropriate cyclic prefix $(\mathrm{CP})$ and restricted number of pilot subcarriers. The interference corrupts the pilot subcarriers utilized for channel estimation and involve the detection process. Initially, the channel covariance matrix and number of channel paths are obtained as least squares estimates of channel on pilot subcarriers. The simulation outcome demonstrates that root mean square error of channel estimate converges with Cramer-Rao Bound (CRB) after some iteration. Furthermore, the BER may arrive enough $\mathrm{CP}$ case; still the delay spread is much larger than $\mathrm{CP}$.

Hakobyan (2017) et.al in [24] has presented a new signal processing strategy for OFDM radar and communication systems that exceeds the OFDM Doppler sensitivity. The Doppler robustness of the proposed strategy open novel viewpoint of system parameterization, enable radar concepts that were previously not feasible. They illustrate simulations that the introduced Doppler correction system is higher to classical signal processing on numerous significant features. The OFDM-MIMO radar measurement prototype was employed for authenticating the presented strategy and displays their efficiency on real-time applications.

Hussein (2019) et.al in [25] has introduced an innovative fully generalized spatial index (FGSI), light-emitting diode (LED) modulation system of MIMO-OFDM optical system. The FGSI was spectrally efficient (SE) visible light communication (VLC) modulation system on LED indices are demoralized on new way for addressing, not just the difficulty of domain configuration of time / frequency of OFDM signal, also give an extra spatial modulation domain (SM). The simulation effects outperform the FGSI by providing superior improvement on BER and Achievable Rate compared with state-of-art OFDM-LED index modulation system. 


\section{Proposed Methodology}

The wave transform is a tool for the signal analysis on time and frequency domain. Beside a variable wave filter, waveforms with dissimilar time and frequency partitions may be designed. Wavelet-based OFDM is easy to execute, flexible to work, and superior orthogonality. The shiftvariant property is considered as a main drawback in Discrete Wavelet Transform (DWT), because it includes complex decimation operation and down sampling. This issue can overcome with another wavelet transform called Redundant Discrete Wavelet Transform (RDWT), which is shift-invariant and thereby it removes complex down sampling operation. Thus due to the redundant property of the RDWT scheme, it is used as a beneficial tool for signal-denoising and statistical signal analysis. The redundancy nature in RDWT scheme makes easier to define rapid changes over various transients. For enhancing the spectral efficiency of MIMO-OFDM system, an innovative method of Multilevel Redundant Discrete Wavelet Transform (ML-RDWT) in a MIMO-OFDM framework is proposed with ICI cancellation by improving the CIR with reduced BER and Inter-symbol-interference (ISI). Here, the weight parameters are optimized by using Optimal Red Deer Algorithm (ORDA).

\subsection{System model of an OFDM framework}

OFDM refers to orthogonal frequency division multiplexing, in which several closely spaced orthogonal subcarrier signals with spectra over each other propagate to carry data on parallel. OFDM is a digital multi-carrier modulation system and because of its advantages, it is more widely utilized on newest high data rate, wide bandwidth wireless applications including Wi-Fi and several cellular telecommunications systems. In OFDM [18], each carrier carries low bit rate data and is therefore more resistant to choose fading, interference, and multipath results. Also, an OFDM scheme provides high degree of spectral efficiency. 


\subsubsection{Architecture model of OFDM system}

An OFDM system typically consists of a transmitter section and receiver section with transmitting and receiving antennas. The system architecture of an OFDM framework is demonstrated on fig. 1 as follows:

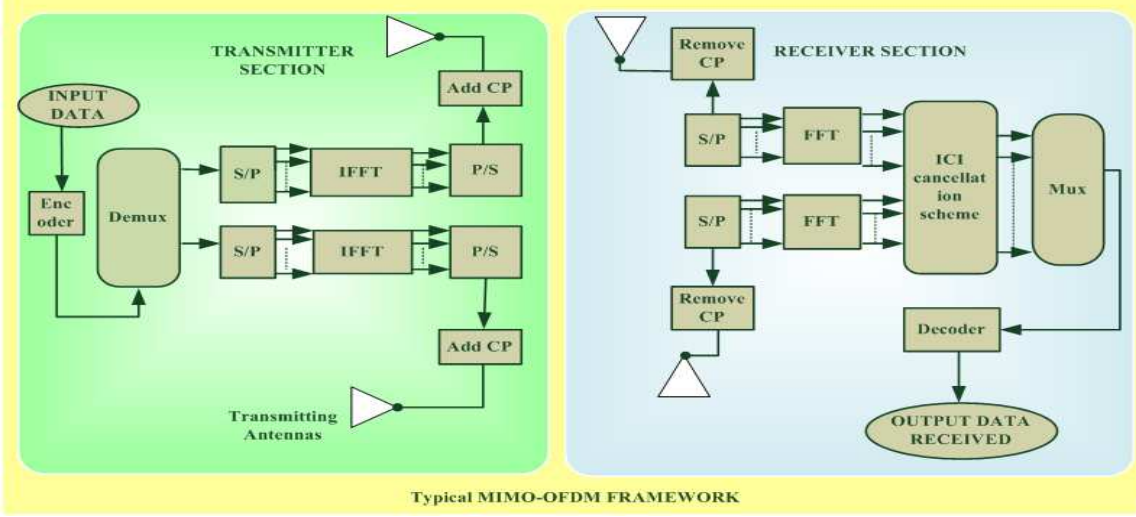

Figure 1: Block diagram for a typical MIMO-OFDM framework

In the above fig, an OFDM system is implemented among two solitary antennas on transmitter and receiver. Here, the modulated OFDM signals coming out from serial-to-parallel converter are denoted by $U_{i}(i=0,1,2, \ldots, K-1)$. These modulated signals have self-governing nature with a random number of variables having zero mean with mean power denoted as $\psi^{2} U$. The signal coming out from IFFT block is expressed as:

$$
u_{y}=\frac{1}{\sqrt{K}} \sum_{i=0}^{K-1} U_{i} e^{j \frac{2 \pi}{K} y i}, y=0,1,2, \ldots ., K-1
$$

The signal coming out from serial-to-parallel converter at the receiver side is denoted by $v_{y}$. Thus, $v_{y}$ may be articulated as:

$$
v_{y}=\left(u_{y} \otimes g_{y}\right) e^{j \frac{2 \pi}{K} y \nabla q t}+s_{y}
$$


In equation (1), the overall number of each subcarrier indicates $K$ and $t$ denotes signal duration. Then, integration of signals from the local oscillator takes place at the receiver. Each signal integrates with an offset frequency those changes from $\nabla q t$ to the frequency of the received signal.

In equation (2), $g_{y}$ implies that impulse channel response, $s_{y}$ denotes AWGN, the symbol $\otimes$ denotes circular convolution and $e^{j \frac{2 \pi}{K} y \nabla q t}$ denotes the spacing ratio frequency of each subcarrier. The FO of the received signal in sampling interval with $\nabla q t$ implies FO with spacing ratio. Also, Cyclic prefix $(\mathrm{CP})$ is added in transmitter end with specific time synchronization.

Then the receiver side, the subcarrier signal in the frequency space coming out from the FFT block is expressed in equation (3) shown below:

$$
\begin{aligned}
& V_{x}=\sum_{i=0}^{K-1} U_{i} I_{i} P(i-x)+s_{x}, x=0,1,2, \ldots, K-1 \\
& =U_{X} I_{X} P(0)+\sum_{i=0}^{K-1} U_{i} I_{i} P(i-x)+s_{x}
\end{aligned}
$$

Therefore, ICI coefficient between $x^{\text {th }}$ and $i^{\text {th }}$ subcarriers are defined by the sequence $P(i-x)$ and it is expressed in the below equation (4) as:

$$
P(i-x)=e^{\left(j \pi(i+v-x)\left(1-\frac{1}{K}\right)\right)} \frac{\sin (\pi(i+v-x))}{K \sin \left(\frac{\pi}{K}(i+v-x)\right)}
$$

In above equation (5), $v$ denotes the normalized FO for each subcarrier.

In equation (3), the first term refers to the transmitted data and the second term refers to ICI caused by subcarriers in OFDM system. Also, the impulse response of channel in frequency domain is expressed $I_{x}$ and $S_{x}$ denotes the frequency space of $s_{y}$.

An extra noise caused by timing jitter will be admitted in the receiver side and therefore equation (3) changes as: 


$$
V_{X}=U_{X} I_{X}+\sum_{i=0, i \neq x}^{K-1} U_{i} I_{i} P\left(z_{i, x}-C\right)+s_{x}
$$

In the above equation, the term $z_{i, x}$ is expressed as

$$
z_{i, x}=\frac{1}{K} \sum_{y=-K / 2+1}^{K / 2} e^{j 2 \pi x \frac{\delta_{y}}{t}} e^{j \frac{2 \pi}{K}(x-i) y}
$$

The parameters $y, x$ and $i$ in the above equation (6) describe the time index, transmitted subcarriers as well as the received subcarriers respectively.

Finally, the digitized signal coming out after dying from the analog-to-digital converter (ADC) block is expressed as:

$$
v_{y}=v\left(y\left(\frac{t}{K}\right)+r_{y}\right)+\alpha_{y}
$$

Then, the CIR is calculated to remove ICI from received signal. Thus, CIR is expressed as below:

$$
C I R=\frac{|P(x)|^{2}}{\sum_{i=0, i \neq x}^{K-1}|P(i-x)|^{2}}
$$

From the above expression, the ICI component is eliminated to achieve improved spectral efficiency. Therefore, (8) is changed as,

$$
C I R=|P(x)|^{2}
$$

Now, the digitized signal received as ADC block is modelled in rate $\frac{R K}{t}$ for accomplishing ultrasampling, here $R$ implies integer value. Thus, capacity of the transmitted signal becomes $\frac{K}{2 t}$ due to the change in the overall $K$ subcarriers in the OFDM framework. The ultra-sampled distinctive time is therefore denoted by $v_{y_{R}}$ and the AWGN indicates $\alpha$. 


$$
v_{y_{R}}=v\left(\frac{y_{R} t}{K R}\right)=\frac{1}{\sqrt{K}} \sum_{x=-K_{L}}^{K_{U}} I_{x} U_{x} e^{\left(\frac{j 2 \pi x}{t} \times \frac{y_{R} t}{K R}\right)}+\alpha\left(\frac{y_{R} t}{K R}\right)
$$

During ultra-sampling, a K-point FFT on receiver is replaced with ultra-sized KR-point FFT. Therefore, received signal from the ADC block changes as follows:

$$
V_{i_{R}}=\frac{1}{\sqrt{K}} \frac{1}{\sqrt{K R}} \sum_{y_{R=-\frac{K R}{2}+1}^{K R / 2}} v_{y_{R}} e^{\left(\frac{-j 2 \pi y i_{R} i_{R}}{K R}\right)}
$$

Thus, the weighting coefficients of extreme model mode can be obtained with combining equations (7), (9) and (10). The weighting coefficients obtained are as follows:

$$
Z_{i_{R}}=\frac{1}{K R} \sum_{y_{R=-\frac{K R}{2}+1}^{K R / 2}}^{j 2 \pi x \frac{\delta_{y_{R}}}{t}}-e^{j \frac{2 \pi}{K R}\left(x-i_{R}\right) y_{R}}
$$

The weight variation of the weight coefficients is provided,

$$
E\left\{\left|z_{i_{R, x}}\right|^{2}\right\} \approx\left(\frac{2 \pi x}{R K t}\right)^{2} \sum_{y_{m}} \sum_{D_{m}} E\left\{r_{y_{R}} r_{p_{R}}\right\}^{j \frac{2 \pi}{R K}(x-i) D_{R}}
$$

When the jitter noise is additive white gaussian noise, then equation (12) can be changed as,

$$
E\left\{\left|z_{i_{R, x}}\right|^{2}\right\} \approx \frac{1}{K R}\left(\frac{2 \pi x}{t}\right)^{2} E\left\{r_{y_{R}}{ }^{2}\right\} x \neq i_{R}
$$

In the above expressions, the symbol $E\{\}$ represents the expectation operation. The term $E\left\{\left|z_{i_{R, x}}\right|^{2}\right\}$ in the above expression (13) indicates the white jitter noise framework. 
From the above expression (13), it is clearly understood that white fluctuation is inversely related with $\mathrm{R}$ value. Thus, by increasing the value of $\mathrm{R}$, can alleviate the result of ISI and ICI caused from result of white noise. .

Finally, the average jitter noise power ${ }^{p_{j}(i)}$ to obtain signal force of ${ }^{i_{\text {th }}}$ subcarrier expressed as,

$$
\begin{aligned}
& \frac{p_{j}(i)}{\psi_{n}{ }^{2}}=\frac{E\left\{\left|\sum_{x}^{K_{u}-K_{i}}\left(z_{i, x}-C_{i, x}\right) U_{x}\right|^{2}\right\}}{\psi_{n}{ }^{2}} \\
& =\sum_{x}^{K_{u}}={ }_{-K_{L}} E\left\{z_{i, x}-\left.C_{i, x}\right|^{2}\right\} \\
& \text { Where, } E\left\{U_{x}{ }^{2}\right\}=\psi_{n}{ }^{2}
\end{aligned}
$$

Thus, the effects of ICI and ISI in the proposed MIMO-ML-RDWT-OFDM framework and their mitigation are explained in the following sections.

\subsection{System architecture of proposed MIMO-ML-RDWT-OFDM framework}

The disadvantages in discrete wavelet transform (DWT) in a MIMO-OFDM framework can be overcome by the use of improved version of redundant discrete wavelet transform (RDWT) called Multi-level redundant discrete wavelet transform (ML-RDWT). Some of the disadvantages faced by a MIMO-OFDM framework due to the use of DWT are: shift-variance deficiency, poor directional selectivity and reduced spectral efficiency. Also, differentiations in wavelet coefficients due to minor shifts on input signal may cause huge differences during energy distribution between the wavelet coefficients at different scales. Therefore, these 
drawbacks should be eliminated to provide better spectral efficiency in many high-speed communication systems.

The system architecture of MIMO-ML-RDWT-OFDM framework is depicted below in fig 2

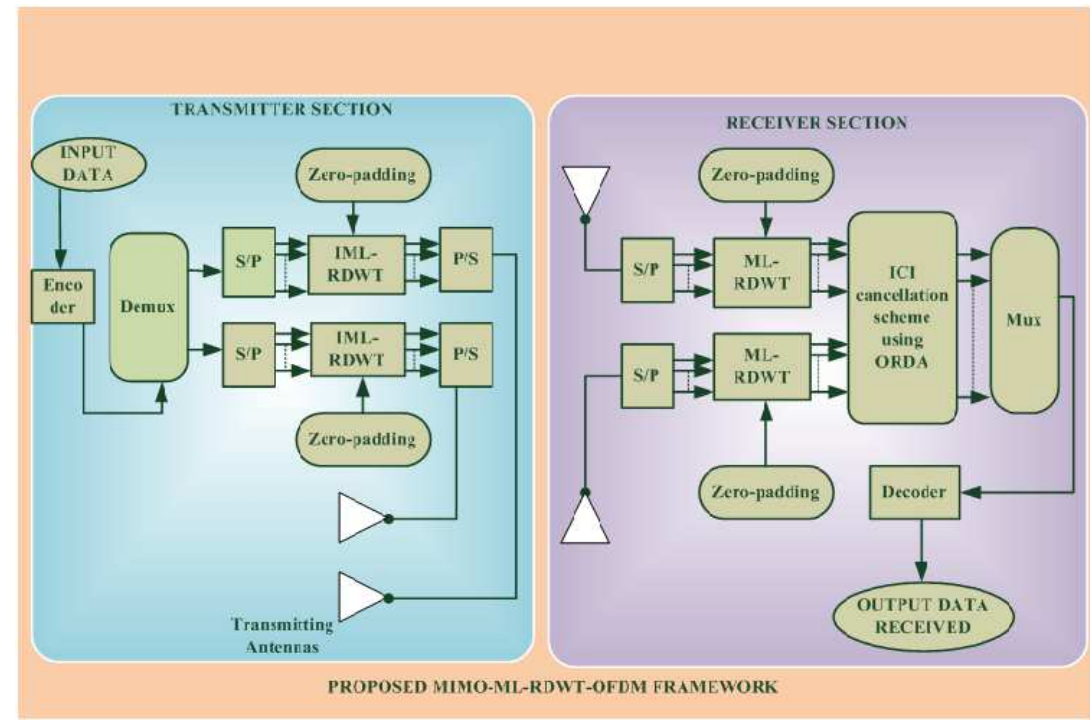

Figure 2: Architecture of proposed MIMO-ML-RDWT-OFDM system

Here, Multi-level redundant discrete wavelet transform (ML-RDWT) is employed to improve the performance of MIMO-OFDM framework through mitigating the effects caused due to ICI, ISI and jitter. The efficiency of the framework is enhanced by lowering the BER values and improving CIR.

The major advantage of using ML-RDWT lies in the fact that, it is shift-invariant due to the elimination of complex down-sampling operation during signal decomposition. The redundancy feature in a MIMO-OFDM framework helps to define the instantaneous changes and transients easier. Also, the redundancy feature establishes full frame expansion and improves the robustness next to additive white Gaussian noise (AWGN) and jitter. Therefore due to the time- 
invariant property and high data rate capability, the ML-RDWT is used as a beneficial tool for many applications like signal-denoising and statistical signal analysis.

Now consider the above fig., in which the received signal coming out from the serial-to-parallel converter block is obtained by the convolution of input signal $u$ from the source and the impulse response $g$ of theinput signal obtained after entering into low-pass filter. Thus, the received signal obtained is expressed as follows:

$$
\begin{aligned}
v[y] & =(u * g)[y] \\
& =\sum_{x=-\infty}^{\infty} u[x] g[y-x]
\end{aligned}
$$

The mathematical expression for output coefficient from the analysis block at level $\mathrm{j}$ of MIMOML-RDWT-OFDM framework is given as:

$$
\begin{aligned}
& a_{j}[x]=\left(a_{j+1}[x] * a_{j}[-x]\right) \\
& d_{j}[x]=\left(d_{j+1}[x] * d_{j}[-x]\right)
\end{aligned}
$$

The mathematical expression for output coefficient from the synthesis block at level $\mathrm{j}$ of MIMOML-RDWT-OFDM framework is given as:

$$
a_{j+1}[x]=\frac{1}{2}\left(a_{j}[x] * A_{j}[x]+d_{j}[x] * D_{j}[x]\right)
$$

In the above expressions, the parameters $A[-x]_{\text {and }} D[-x]_{\text {denotes low and high-pass filter }}$ coefficient during analysis. $A[x]$ and $D[x]$ implies low and high-pass filter coefficient during synthesis. 
In the proposed MIMO-ML-RDWT-OFDM-ORDA framework, the cyclic prefix (CP) block is not added in the transmitter section. The cyclic prefix produces loss of spectral efficiency during data transmission. The modulated signal coming out from the serial-to-parallel converter block is transmitted using Zero padding (ZP). ZP strategy is extensively used in DWT systems to compute highly interpolated spectra of the zero-padded signal. Adding ZP effect will help to extend a signal with zeros.

The CIR for ICI cancellation may be estimated with help of expression shown below:

$$
C I R=\frac{|-\eta P(2 x+1-K)+(1+\mu \eta) P(0)-\mu P(K-1-2 x)|^{2}}{\sum_{i=0, i \neq x}^{\frac{K}{2}-1}|-\eta P(i-K+x+1)-P(i-x)-\mu P(K-1-i-x)+\eta \mu P(i-x)|^{2}}
$$

From the above expression, the ICI/ISI component is removed to achieve improved spectral efficiency. Thus, the above equation can be rewritten as,

$$
C I R=|-\eta P(2 x+1-K)+(1+\mu \eta) P(0)-\mu P(K-1-2 x)|^{2}
$$

In the above expressions, the parameters $\quad \eta$ and $\mu$ represents the optimized weights. The optimality of these weights depends on the frequency estimation factor, which are difficult for time varying channels. The existing MIMO-OFDM systems requires more time to compute the optimal weights. Thus, there arises a need for complex hardware design and this leads to the use expensive hardware's. Therefore to reduce the computation time and complexity in design, an optimal ML-RDWT technique is employed in the proposed work. 


\subsection{Optimal weights computation using ORDA}

The weight parameters are efficiently optimized in the proposed approach by using an optimal red deer algorithm (ORDA), an improved version of red deer algorithm (RDA).

\subsubsection{Optimal red deer algorithm (ORDA)}

The meta-heuristic algorithms used in the existing methods faces pre-mature convergence and do not optimize the weight parameters efficiently in providing better performance. Therefore, a novel meta-heuristic based optimization approach named optimal red deer algorithm (ORDA), an improved version of red deer algorithm (RDA) is employed in the proposed work to optimize the weight parameters $\rho, \eta$ and $\mu$ efficiently by obtaining optimal best solution with improvement in CIR value, thereby achieving better performance.

The RDA begins with primary population known as red deer (RD). Among the population, the number of best RD is separated into two kinds: "hinds" and "male RDs". Also, a harem is a group of RD women. The common steps of this evolutionary algorithm are assumed with competition of male RDs to obtain the harem with more hinds through roaring and fighting behaviours. Depending upon the roaring power, the male RDs are separated into two groups, namely the commanders and stags. Harems are made up of commanders. The number of hinds on harems depends on commander ability during roaring and fighting. The below fig 3 illustrates the flowchart model of ORDA. 


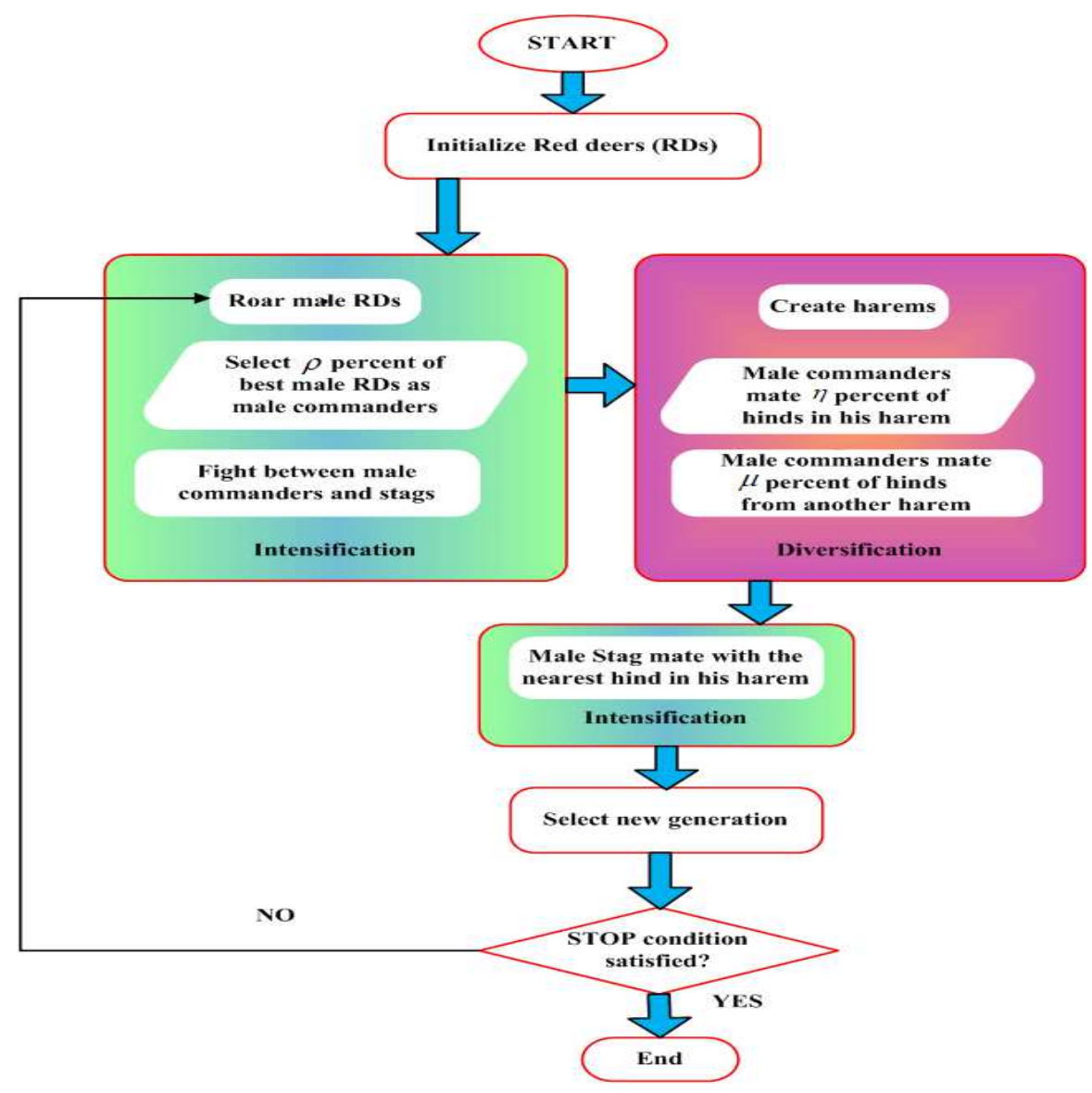

Figure 3: Flowchart model for ORDA

Consider the above figure, in which there are three phases namely: the exploitation phase, the exploration phase and mating phase. Through the exploitation phase, the roaring of male RD serves as a counterpart during local search on space for solution. Here, the fighting process among commanders and stags is assumed from a local search. During the exploration phase based on the power of the harems, they are distributed to the commanders. Here, the commander of harem mates through a percentage of hinds on harem and hinds in another harem. During breeding season, a stag mates with its closest hind based on minimal distance, regardless of harem limitation. The third important phase in ORDA is the mating phase. As a result of mating 
process, new offspring of RDs will be produced. In ORDA, a user can able to tune the exploration and the exploitation phase based on their requirements.

Also, there exist two important sections in ORDA. The first section refers to the intensification section and the second section refers to the diversification section. The parameters $\eta, \mu$ and $\rho$ are employed in optimal red deer algorithm for controlling the exploration and exploitation phases. The parameter $\rho$ control the intensification phase, while the parameters $\eta$ and $\mu$ control a diversification phase.

At last, the most suitable value $\rho, \eta$ and $\mu$ can be obtained according to CIR turn-off condition. Therefore, improved CIR of is obtained in our proposed approach.

\subsubsection{Steps in ORDA}

The steps in the optimal red deer algorithm (ORDA) are explained below as follows:

\section{Step 1: Generate initial RDs}

Initialize RDs by selecting some random points on the functions. In this step, we select the best RDs as male RDs and the remaining RDs are considered as hinds. $R D=\left\lfloor U_{1}, U_{2}, \ldots \ldots \ldots, U_{K_{\text {Var }}}\right\rfloor$

$$
q(R D)=q\left[U_{1} U_{2}, \ldots \ldots . ., U_{K_{\text {Var }}}\right\rfloor
$$

\section{Step 2: Roar male RDs}

The roaring process decides the capability of male RDs. Here, each male RDs changes their position.

\section{Step 3: Select the male RDs as male commander}

In this step, $\rho \%$ of better male RDs is chosen as male commanders.

$$
\begin{aligned}
& K_{\text {male com }}=\operatorname{round}\left\{\rho K_{\text {male }}\right\} \\
& K_{\text {stag }}=K_{\text {male }}-K_{\text {male com }}
\end{aligned}
$$




\section{Step 4: Fight between male commanders and stags}

In this step, every male commander randomly fights with the stags. Here, best objective function is selected that is much better than the prior ones.

\section{Step 5: Form a group of best female harems}

The harems are formed by the male commanders.

$$
\begin{aligned}
& B_{y}=b_{y}-\max \left\{b_{l}\right\} \\
& p_{y}=\left|\frac{B_{y}}{\sum_{l=1}^{N_{\text {male com }}} b_{l}}\right| \\
& \text { Kharem } \\
& \text { horound }\left\{p_{y} K_{\text {hind }}\right\}
\end{aligned}
$$

\section{Step 6: Mating process}

In this step, the male commanders of the harem mate with $\eta \%$ of hinds in his harem. Then the male commanders of the harem mate with $\mu \%$ of hinds from another harem.

$$
\begin{aligned}
& \text { Kharem }_{y}^{\text {mate }}=\operatorname{round}\left\{\eta{\text { K } \left.\text { harem }_{y}\right\}}^{\text {harem }}{ }^{\text {mate }}=\operatorname{round}\left\{\mu{\text { K } \left.\text { harem }_{y}\right\}}^{\text {hava }}\right\}\right.
\end{aligned}
$$

\section{Step 7: Mate stag with the nearest hind}

In this phase, every stag mate with the nearest hind. During breeding season, each male RDs select the best handy hind among a group of hinds. This best selected hind may be in his harem or from another harem.

$$
D_{l}=\left(\sum_{j \in J}\left(\operatorname{stag}_{j}-\text { hind }_{j}{ }^{l}\right)^{2}\right)^{1 / 2}
$$




\section{Step 8: Select the new generation}

here select the best male RDs for forming a new generation by considering the best suitable fitness value and then select hinds for the new generation.

\section{Step 9: Convergence or stopping condition}

In this step, it can stop the iteration when reaching the best solution.

The behaviour of the ORDA in finding the best solution is better than the traditional RDA [15], GA [26] and PSO [27].

\section{Simulation Results}

In this section a simulation analysis is done for clarifying the efficiency of the proposed MIMOML-RDWT-OFDM-ORDA system during high speed data transmission that requires increased spectral efficiency. The simulation parameters are explained on Table 1 as follows:

Table 1:Simulation Parameters

Parameter

No. of sub carriers

No. of symbols

Frequency offset (FO)

Channel model

FFT/IFFT size

Modulation system

Constellation points

Cyclic prefix (CP)
DFT-OFDM

512

2000

0.15 and 0.25

AWGN

512

QAM and QPSK

$4,8,16, \ldots \ldots .$. and so on

\section{ML-RDWT-OFDM}

512

2000

0.15 and 0.25

AWGN with Rayleigh and rician fading

environment

QAM and QPSK

$4,8,16, \ldots \ldots .$. and so on

No CP 
Transmitted power

Bandwidth

performance
1 watt

$1 \mathrm{~b} / \mathrm{s} / \mathrm{Hz}$
1 watt

$10 \mathrm{~b} / \mathrm{s} / \mathrm{Hz}$

\subsection{Performance Evaluation}

In the proposed work, a novel MIMO-OFDM system based on multi-level redundant discrete wavelet transform with ORDA is suggested for diminishing the effect caused by ICI and ISI. The proposed MIMO-ML-RDWT-OFDM framework is simulated using MATLAB platform on windows 2007 system by Intel (R) Core (TM) i7-4790 3.6 GHz CPU with 8 GB of RAM. In this work, the BER, ISI and ICI of MIMO-ML-RDWT-OFDM framework is compared to BER, ISI and ICI in the existing methods like MIMO-DWT-OFDM-RDA [44], MIMO-RNS-OFDMPNMA [41], MIMO-OFDM-BMA [42] and MIMO-OFDM-ICIMA [43].

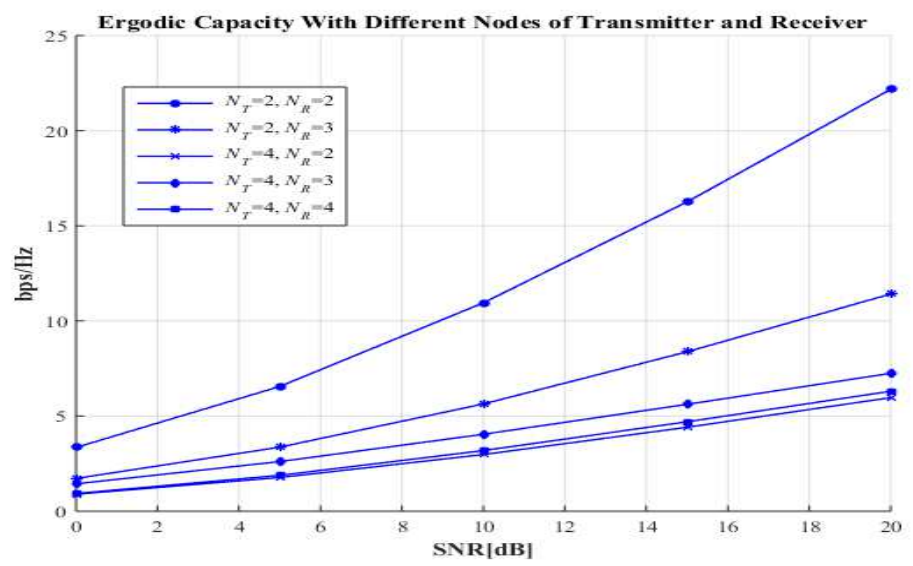

Figure 4: Ergodic capacity for difference nodes of transmitter and receiver

The above fig.4 illustrates the variation of Ergodic capacity for difference nodes of transmitter and receiver. From figure 4, it is noted that the Ergodic capacity increases as the SNR increases for difference nodes of transmitter and receiver. 


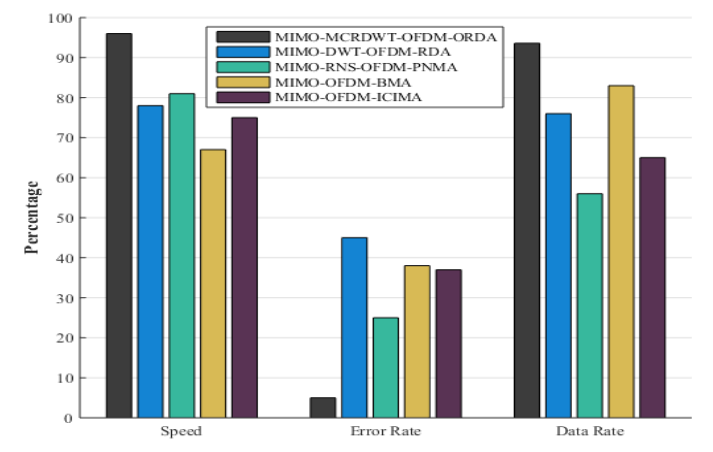

Figure 5: Comparison of speed, error rate and data rate of the proposed method with different existing methods

The above fig.5 illustrates the percentage comparison of speed, error rate and data rate of the proposed MIMO-ML-RDWT-OFDM-ORDA method with various existing methods like MIMODWT-OFDM-RDA, MIMO-RNS-OFDM-PNMA, MIMO-OFDM-BMA and MIMO-OFDMICIMA. From the above fig. 5, it is clearly understood that the proposed method MIMO-MLRDWT-OFDM-ORDA achieves speed of $23 \%, 19 \%, 42 \%$ and $29 \%$, which is higher when compared to the speed in the existing methods. The proposed method achieves high data rate of about $25 \%, 68 \%, 15 \%$ and $45 \%$ when compared to the above mentioned existing techniques. Also, the proposed method produces error rate of $89 \%, 80 \%, 87 \%$ and $86 \%$, which is very low when comparing to the other existing methods.

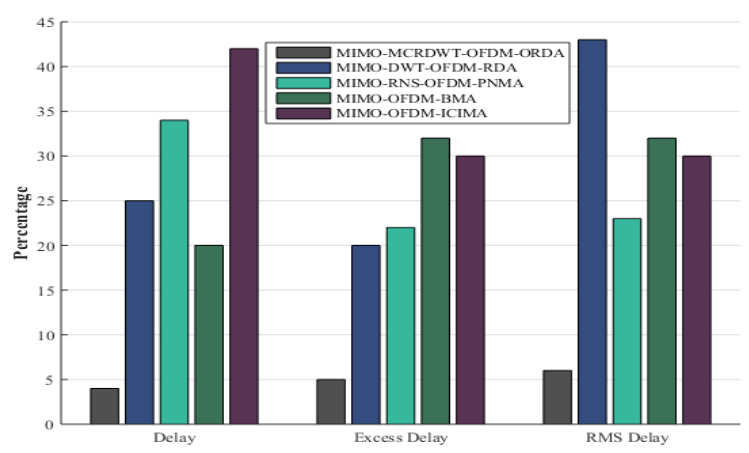

Figure 6: Comparison of various types of delay's in proposed method to that of existing methods 
From fig. 6, it is clearly noted that the various kinds of delay's like excess delay and RMS delay on proposed system is very low compared with delay's in existing methods. The average delay in proposed system is about $84 \%, 88 \%, 80 \%$ and $90 \%$, which is very low when compared to the average delay in the existing methods. The excess delay in the proposed method is about $75 \%, 77 \%, 84 \%$ and $83 \%$, which is very low when compared to the excess delay in the existing methods. The RMS delay in the proposed method is about $86 \%, 74 \%, 81 \%$ and $80 \%$, which is very low when compared to the RMS delay in the existing methods.

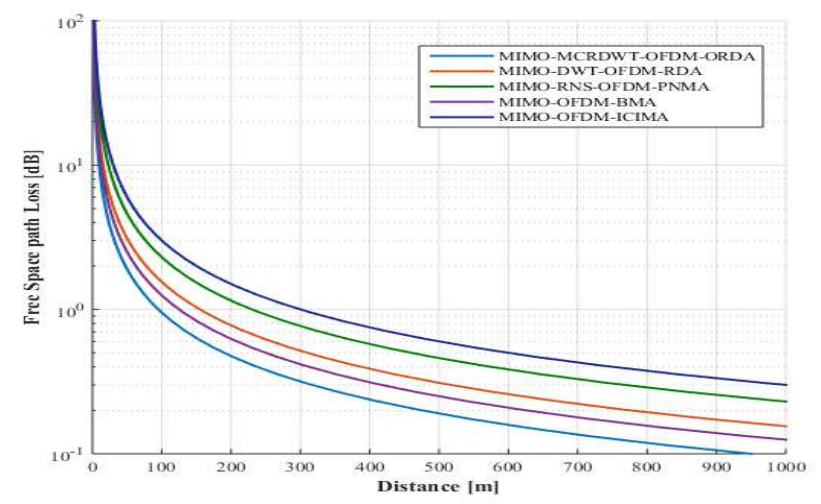

Figure 7: Comparison of Free space path loss for different distances

From the above fig.7, it is clearly understood that the free space path loss for the proposed approach decreases as the distance increases. Thus, the proposed method possesses less free space path loss, when compared to the other existing approaches.

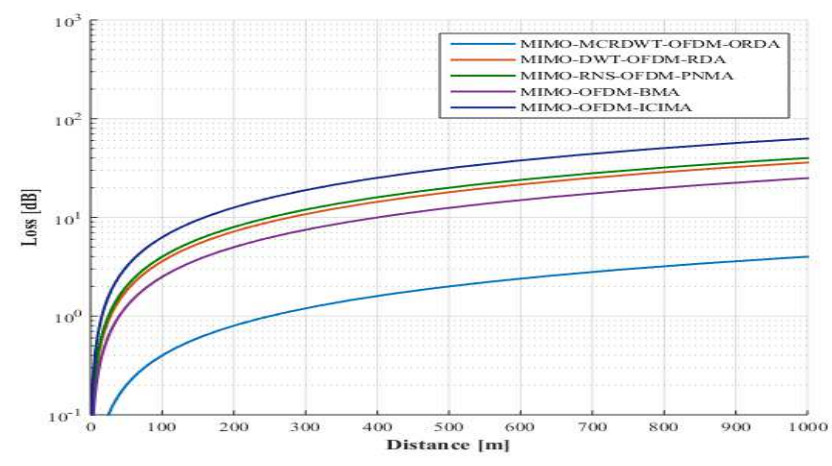

Figure 8: Comparison of loss for different distances 
From the above fig. 8, it is noted that the loss in the proposed approach decreases when the distance increases. Thus, the proposed approach achieves reduced loss while compared with other existing systems.

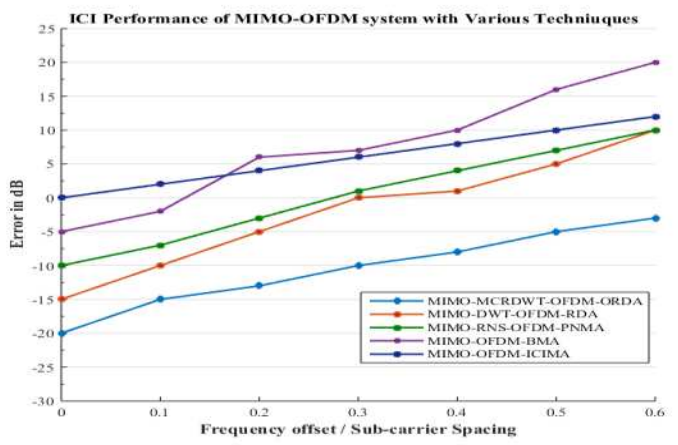

Figure 9: Comparison of error in proposed method and existing methods for different $\mathrm{FO}$ values

The above fig.9 illustrates the ICI performance in the proposed MIMO-ML-RDWT-OFDMORDA system with various existing techniques compared. From figure, it observed that proposed system exhibits less ICI, while compared with other existing systems.

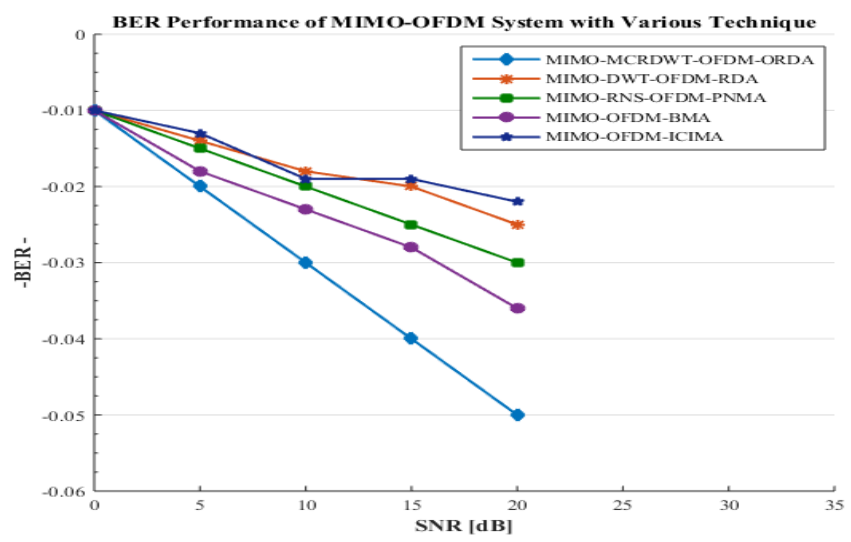

Figure 10: Comparison of BER and SNR in the proposed approach to that of the existing approaches.

From fig.10, it obviously observed that the BER in proposed approach decreases even when the SNR increases. 


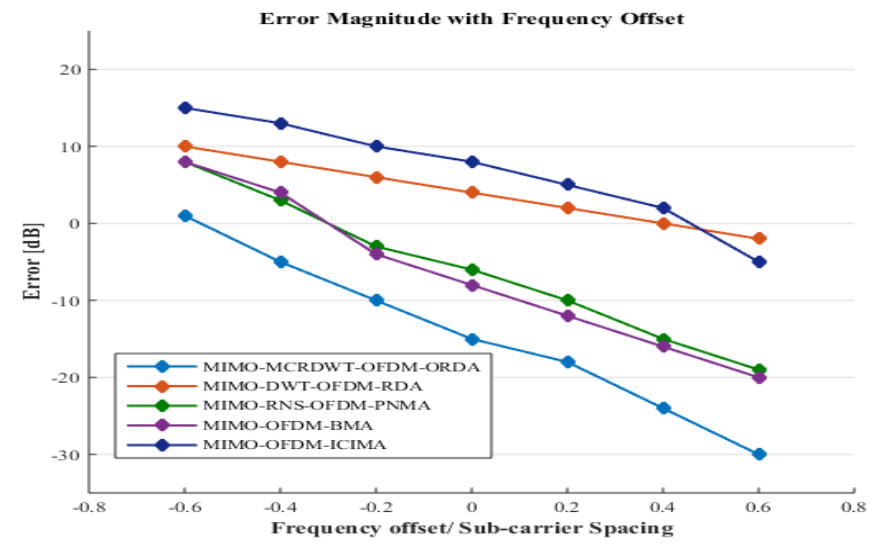

Figure 11: Comparison of error in the proposed method with various existing methods for different $\mathrm{FO}$ values.

From the above fig.11, it is observed that the proposed method exhibits less error when compared to the other exiting techniques.

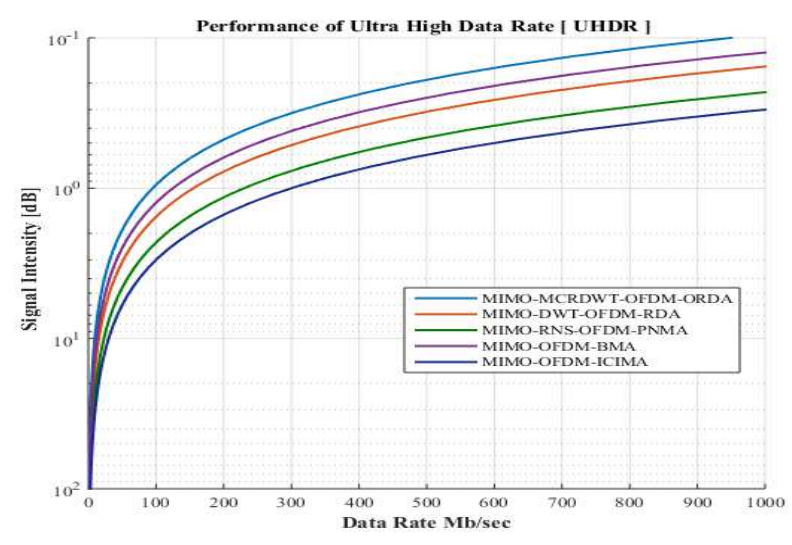

Figure 12: Comparison of signal intensity for varying data rates

The above fig.12 depicts the comparison of signal intensities for different data rates. From fig. 12 , it is observed that proposed system achieves increased signal intensity even when the data rate increases. Thus, the proposed method possesses high signal intensity while compared with other existing systems. 


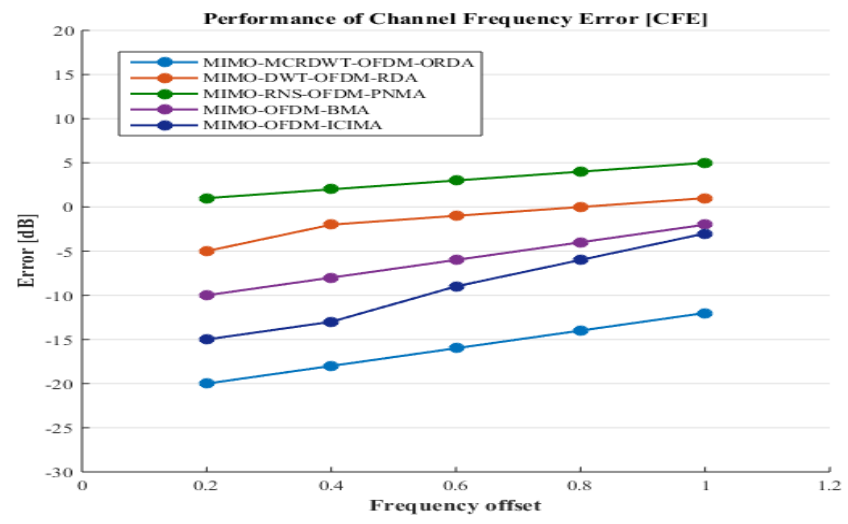

Figure 13: Comparison of channel frequency error for different FO values

From the fig. 13, it is noted that the channel frequency error (CFE) in the proposed approach decreases for different FO values. Thus, the proposed method possesses less CFE when compared to CFE in the exiting approaches.

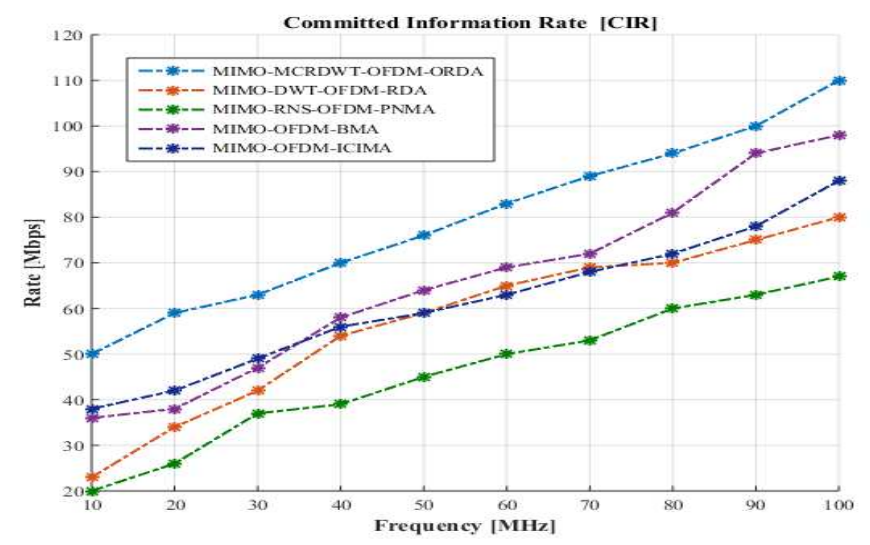

Figure 14: Comparison of Carrier-to-interference power ratio for different frequency values

The above fig.14 depicts the CIR achieved through proposed approach to that of the CIR in the exiting approaches for different frequencies. From the fig, it is observed that the proposed method achieves high CIR when compared to the exiting approaches. 


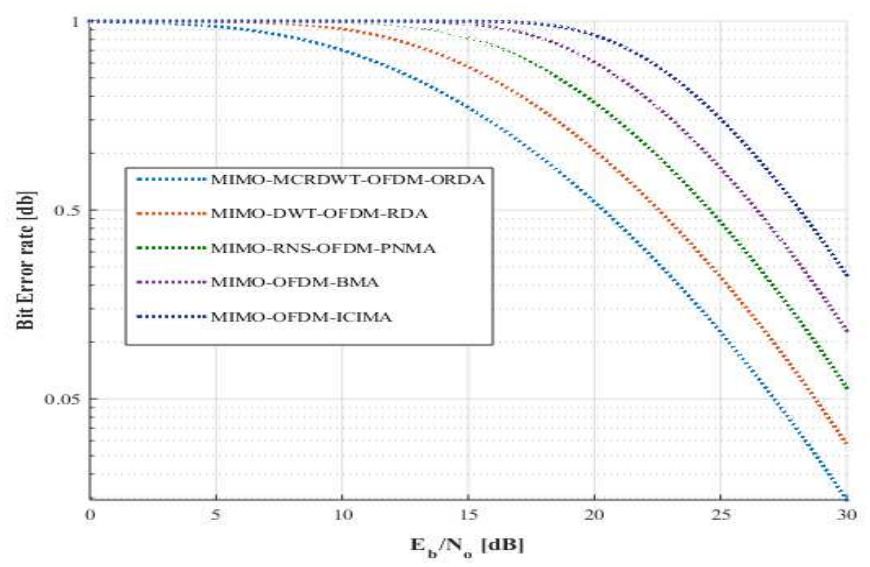

Figure 15: Comparisonof BER in the proposed approach to that of existing approaches

The above fig.15 illustrates the BER comparison of the proposed approach to that of the exiting approaches. From fig.15, it observed that proposed method achieves decreased BER while compared with other exiting methods.

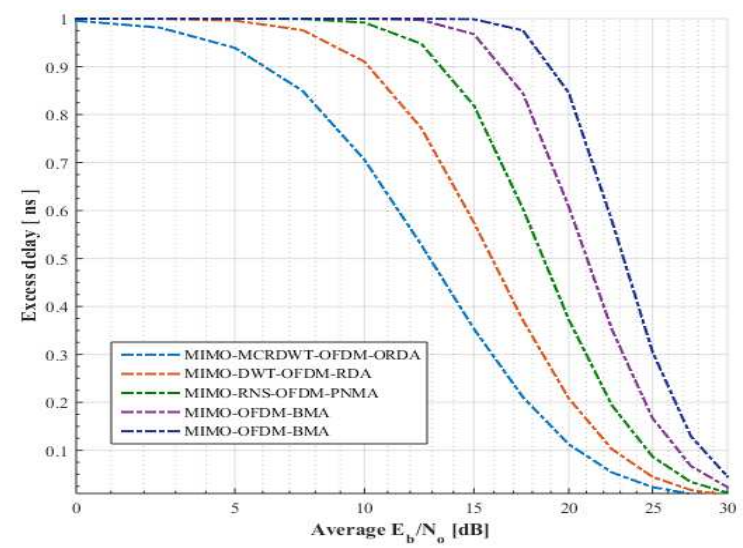

Figure 16: Comparison of excess delay in proposed method to that of the excess delay from various exiting methods

The above fig.16 illustrates the comparison of excess delay from the proposed method to that of excess delay from the existing methods. From figure, it is obviously noted that that excess delay exhibited by proposed system is minimum when compared to the excess delay in the existing methods. 


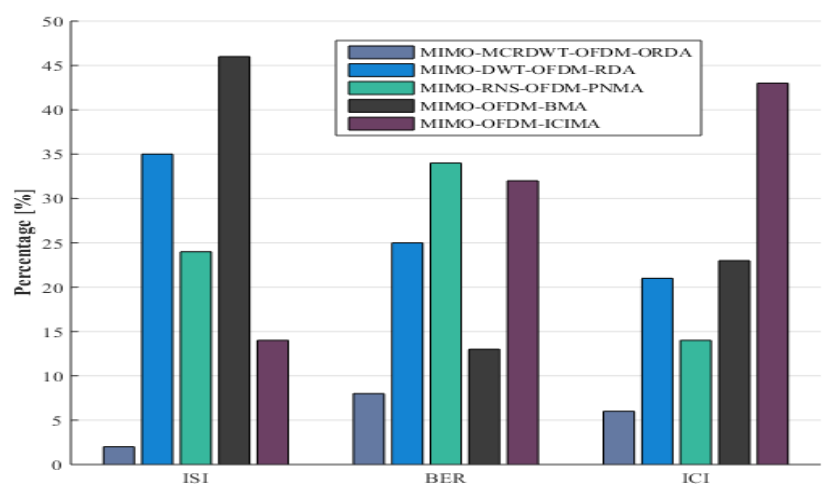

Figure 17: Comparison of ISI, BER and ICI in the proposed approach to that of the existing approaches

From the above fig.17, it is clearly understood that the ISI, ICI and BER of proposed technique is minimum when compared with ISI, ICI and BER in the existing techniques. The proposed method exhibits ISI of $94 \%, 91 \%, 95 \%$ and $86 \%$, which is minimal while compared with existing techniques. The BER in the proposed method is $68 \%, 76 \%, 38 \%$ and $75 \%$ minimal while compared with BER in the existing techniques. The ICI in the proposed method is $71 \%$, $57 \%, 73 \%$ and $86 \%$ less while compared with ICI in the existing techniques. Therefore, the efficiency of the proposed system MIMO-ML-RDWT-OFDM-ORDA is much better when compared to the other existing MIMO-OFDM approaches.

\section{Conclusion}

In this work, a novel multi-level redundant discrete wavelet transform in MIMO-OFDM framework to improve the spectral efficiency during high-speed data transmission is proposed. Here, the effects caused by ICI, ISI and BER have been mitigated with increased CIR. In this, a MIMO-OFDM system with RDWT is executed that improves spectral performance and does not need any cyclic prefix (CP). Also, an optimal red deer algorithm is employed for optimizing weight parameters and mitigates the effects caused by ISI, ICI and BER by improving CIR in an efficient way. Therefore, the proposed scheme MIM0-ML-RDWT-OFDM-ORDA scheme is 
highly suitable for use during high-speed data transmission in mobile communication systems with improved spectral efficiency. From the simulation results, it is clearly identified that our proposed MIM0-ML-RDWT-OFDM-ORDA method possesses low BER of 68\%, 76\%, 38\% and 75\% when compared to the existing methods like MIMO-DWT-OFDM-RDA, MIMO-RNSOFDM-PNMA, MIMO-OFDM-BMA and MIMO-OFDM-ICIMA. The proposed MIM0-MLRDWT-OFDM-ORDA method possesses low ISI of $94 \%, 91 \%, 95 \%$ and $85 \%$ when compared to the existing methods described. Also, the proposed MIM0-ML-RDWT-OFDM-ORDA method possesses low ICI of $71 \%, 57 \%, 74 \%$ and $86 \%$ when compared to the existing methods mentioned above. Thus, the proposed method achieves improved spectral efficiency with increase in CIR performance.

\section{Data Availability Statement}

Data sharing is not applicable to this article as no new data were created or analyzed in this study.

\section{Declaration of Statement}

The authors declare that they have no known competing financial interests or personal relationships that could have appeared to influence the work reported in this paper

\section{References}

[1] Ylioinas, J., \& Juntti, M. (2008). Iterative joint detection, decoding, and channel estimation in turbo-coded MIMO-OFDM. IEEE Transactions on Vehicular Technology, 58(4), 17841796.

[2] Lu, B., Yue, G., \& Wang, X. (2004). Performance analysis and design optimization of LDPC-coded MIMO OFDM systems. IEEE Transactions on signal processing, 52(2), 348361. 
[3] Hwang, T., Yang, C., Wu, G., Li, S., \& Li, G. Y. (2008). OFDM and its wireless applications: A survey. IEEE transactions on Vehicular Technology, 58(4), 1673-1694.

[4] Liu, J., \& Li, J. (2005). Turbo processing for an OFDM-based MIMO system. IEEE Transactions on Wireless Communications, 4(5), 1988-1993.

[5] Ganesh, R. S., Jayakumari, J., \& Akhila, I. P. (2011). Channel estimation analysis in MIMO-OFDM wireless systems. In 2011 International Conference on Signal Processing, Communication, Computing and Networking Technologies (pp. 399-403). IEEE.

[6] Akhtman, J., \& Hanzo, L. (2007). Advanced channel estimation for MIMO-OFDM in realistic channel conditions. In 2007 IEEE International Conference on Communications (pp. 2528-2533). IEEE.

[7] Qiao, X., Cai, Y., \& Xu, Y. (2005). Joint iterative decision feedback channel estimation for turbo coded V-BLAST MIMO-OFDM systems. In IEEE International Symposium on Communications and Information Technology, 2005. ISCIT 2005. 2, 1384-1388. IEEE.

[8] Vidhya, K., \& Kumar, K. S. (2014). An Intelligent Channel Estimation Approach for MIMO-OFDM Systems using Meta-heuristic Optimization Algorithm. Research Journal of Applied Sciences, Engineering and Technology, 7(19), 4079-4087.

[9] Gao, Z., Dai, L., Lu, Z., Yuen, C., \& Wang, Z. (2014). Super-resolution sparse MIMOOFDM channel estimation based on spatial and temporal correlations. IEEE communications letters, 18(7), 1266-1269.

[10] He, R., Ai, B., Stüber, G. L., Wang, G., \& Zhong, Z. (2017). Geometrical-based modeling for millimeter-wave MIMO mobile-to-mobile channels. IEEE Transactions on Vehicular Technology, 67(4), 2848-2863. 
[11] Guan, K., Ai, B., Fricke, A., He, D., Zhong, Z., Matolak, D. W., \& Kürner, T. (2016). Excess propagation loss of semi-closed obstacles for inter/intra-device communications in the millimeter-wave range. Journal of Infrared, Millimeter, and Terahertz Waves, 37(7), 676-690.

[12] Barazzetta, M., Micheli, D., Bastianelli, L., Diamanti, R., Totta, M., Obino, P., \& Primiani, V. M. (2017). A comparison between different reception diversity schemes of a 4G-LTE base station in reverberation chamber: a deployment in a live cellular network. IEEE Transactions on Electromagnetic Compatibility, 59(6), 2029-2037.

[13] Recanatini, R., Moglie, F., \& Primiani, V. M. (2013). Performance and immunity evaluation of complete WLAN systems in a large reverberation chamber. IEEE transactions on electromagnetic compatibility, 55(5), 806-815.

[14] Mounika, N., Rani, M. D., Narayana, J. L., \& Kalyani, M. N. L. (2019). ICI Cancellation in OFDM Systems Under Stanford University Interim Channel Model. In Innovations in Electronics and Communication Engineering, 241-248. Springer, Singapore.

[15] Fathollahi-Fard, A. M., Hajiaghaei-Keshteli, M., \& Tavakkoli-Moghaddam, R. (2020). Red deer algorithm (RDA): a new nature-inspired meta-heuristic. Soft Computing, 1-29.

[16] Sharief, A. H., \& Sairam, M. S. (2019). Performance analysis of MIMO-RDWT-OFDM system with optimal genetic algorithm. AEU-International Journal of Electronics and Communications, 111, 152912.

[17] Khalifa, M. A., Emam, A. E., \& Youssef, M. I. (2019). ICI and PAPR enhancement in MIMO-OFDM system using RNS coding. In 2019 IEEE Jordan International Joint Conference on Electrical Engineering and Information Technology (JEEIT) (pp. 7-12). IEEE. 
[18] Nakamura, A., Otsubo, H., \& Itami, M. (2018). A Study on Dual-Polarized MIMO-ICI Canceller With Complexity Reduction Under Mobile Reception of OFDM Signals. IEEE Transactions on Broadcasting, 65(3), 589-600.

[19] Lu, J., Chen, X., Liu, S., \& Fan, P. (2017). Location-aware ICI reduction in MIMO-OFDM downlinks for high-speed railway communication systems. IEEE Transactions on Vehicular Technology, 67(4), 2958-2972.

[20] Hao, J., Wang, J., \& Pan, C. (2016). Low complexity ICI mitigation for MIMO-OFDM in time-varying channels. IEEE Transactions on Broadcasting, 62(3), 727-735.

[21] Fowler, J. E. (2005). The redundant discrete wavelet transform and additive noise. IEEE Signal Processing Letters, 12(9), 629-632.

[22] Paek, M. J., Kim, W. C., Kim, M. Y., \& Song, H. K. (2019). Spatial Phase Coding With CoMP for Performance Enhancement Based on MIMO-OFDM in HetNet System. IEEE Access, 7, 62240-62250.

[23] Pham, T., Le-Ngoc, T., Woodward, G. K., \& Martin, P. A. (2016). Channel estimation and data detection for insufficient cyclic prefix MIMO-OFDM. IEEE Transactions on Vehicular Technology, 66(6), 4756-4768.

[24] Hakobyan, G., \& Yang, B. (2017). A novel intercarrier-interference free signal processing scheme for OFDM radar. IEEE Transactions on Vehicular Technology, 67(6), 5158-5167.

[25] Hussein, H. S., \& Hagag, M. (2019). Optical MIMO-OFDM with fully generalized indexspatial LED modulation. IEEE Communications Letters, 23(9), 1556-1559.

[26] Raut, S. N., \& Jalnekar, R. M. (2019). Hybrid optimisation for priority-based scheduling in multi-user STBC-MIMO-OFDM. IET Communications, 13(20), 3391-3400. 
[27] Ali, W., Li, Y., Tanoli, S. A. K., \& Raja, M. A. Z. (2020). Integrated swarming computing paradigm for efficient estimation of channel parameters in MIMO system. Wireless Personal Communications, 115(1), 77-102.

[28] Shahbaztabar, D., Alirezaee, S., Ahmadi, M., \& Heydari, R. (2018). A MC-CDMA system based on orthogonal filter banks of wavelet transforms and partial combining. $A E U$ International Journal of Electronics and Communications, 94, 128-138.

[29] He, R., Ai, B., Stüber, G. L., Wang, G., \& Zhong, Z. (2017). Geometrical-based modeling for millimeter-wave MIMO mobile-to-mobile channels. IEEE Transactions on Vehicular Technology, 67(4), 2848-2863.

[30] He, R., Li, Q., Ai, B., Geng, Y. L. A., Molisch, A. F., Kristem, V., \& Yu, J. (2017). A kernel-power-density-based algorithm for channel multipath components clustering. IEEE Transactions on Wireless Communications, 16(11), 7138-7151.

[31] Fan, W., Carton, I., Kyosti, P., Karstensen, A., Jamsa, T., Gustafsson, M., \& Pedersen, G. F. (2016). A Step Toward 5G in 2020: Low-cost OTA performance evaluation of massive MIMO base stations. IEEE Antennas and Propagation Magazine, 59(1), 38-47.

[32] Ai, B., Guan, K., He, R., Li, J., Li, G., He, D., \& Huq, K. M. S. (2017). On indoor millimeter wave massive MIMO channels: Measurement and simulation. IEEE journal on selected areas in communications, 35(7), 1678-1690.

[33] Li, Y., Jiang, H., Yu, Y., \& Du, S. (2016). Efficient inter-carrier interference cancellation transmissions for cooperative networks with frequency offsets. IET Communications, 10(18), 2575-2581.

[34] Ma, T. (2017). A new inter-carrier interference self-cancellation mapping scheme over fast time-varying fading channels. Electronics Letters, 53(21), 1433-1435. 
[35] Maya, D. P., \& Kandiyil, A. C. (2016). Blind inter carrier interference compensation in MIMO SC-IFDMA system using firefly algorithm. AEU-International Journal of Electronics and Communications, 70(6), 857-865.

[36] Ali, N., Youssef, M. I., \& Tarrad, I. F. (2020). ICI reduction by parallel concatenated encoder using wavelet transforms. In Information and Communication Technology for Sustainable Development (pp. 415-421). Springer, Singapore.

[37] Sarowa, S., Kumar, N., Agrawal, S., \& Sohi, B. S. (2020). Evolution of PAPR reduction techniques: A wavelet based OFDM approach. Wireless Personal Communications, 115(2), $1565-1588$.

[38] Ayeswarya, R. (2019). Fractional wavelet transform based OFDM system with cancellation of ICI. Journal of Ambient Intelligence and Humanized Computing, 1-11.

[39] Dogani, A., Dourandish, A., Ghorbani, M., \& Shahbazbegian, M. R. (2020). A Hybrid Meta-Heuristic for a Bi-Objective Stochastic Optimization of Urban Water Supply System. IEEE Access, 8, 135829-135843.

[40] Zhou, B., \& He, Z. (2020). A material handling scheduling method for mixed-model automotive assembly lines based on an improved static kitting strategy. Computers \& Industrial Engineering, 140, 106268.

[41] Khalifa, M. A., Emam, A. E., \& Youssef, M. I. (2019). ICI and PAPR enhancement in MIMO-OFDM system using RNS coding. In 2019 IEEE Jordan International Joint Conference on Electrical Engineering and Information Technology (JEEIT) (pp. 7-12). IEEE. 
[42] Liu, H., Duan, Z., \& Chen, C. (2020). Wind speed big data forecasting using time-variant multi-resolution ensemble model with clustering auto-encoder. Applied Energy, 280, 115975.

[43] Djebbar, F., \& Abu-Ali, N. (2017). Lightweight noise resilient steganography scheme for Internet of Things. In GLOBECOM 2017-2017 IEEE Global Communications Conference (pp. 1-6). IEEE.

[44] Liu, X., Liu, Y., Wang, X., \& Zhou, J. (2017). Application of communication OFDM waveform to SAR imaging. In 2017 IEEE Radar Conference (RadarConf) (pp. 17571760). IEEE. 
Figures
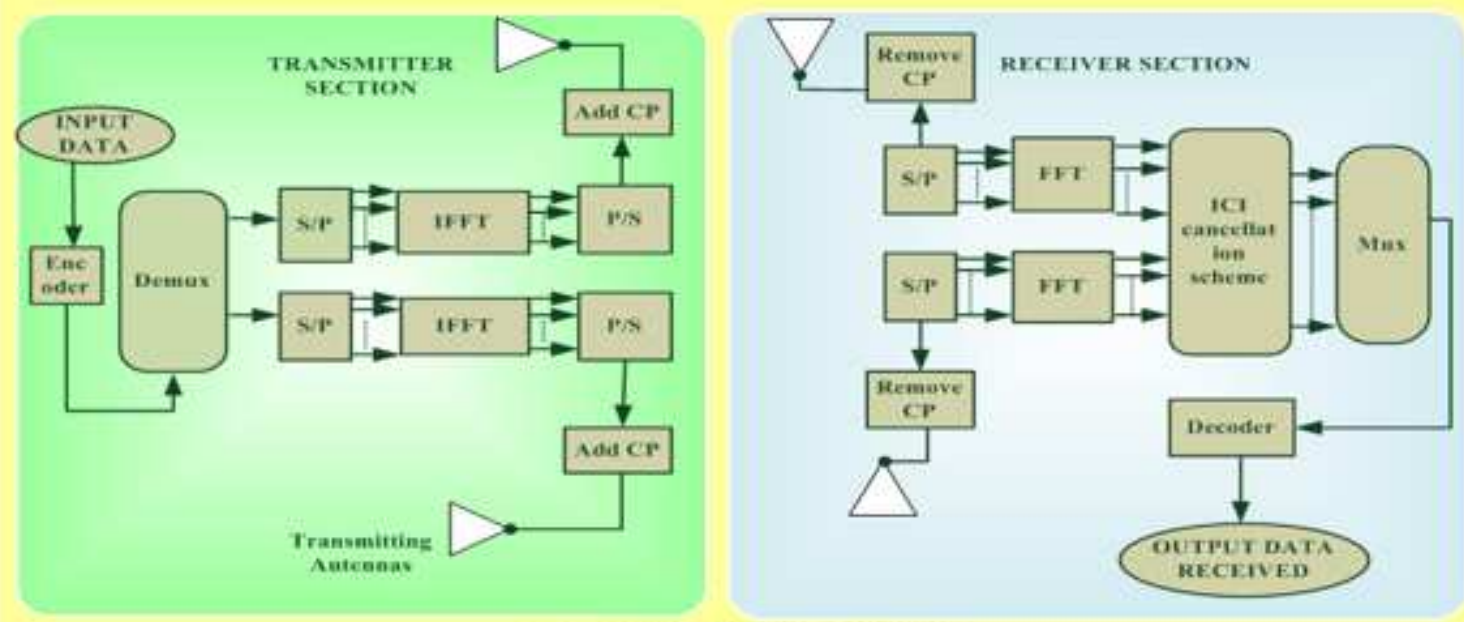

TypicaI MIMO-OFDM FRAMEWOKK

\section{Figure 1}

Block diagram for a typical MIMO-OFDM framework

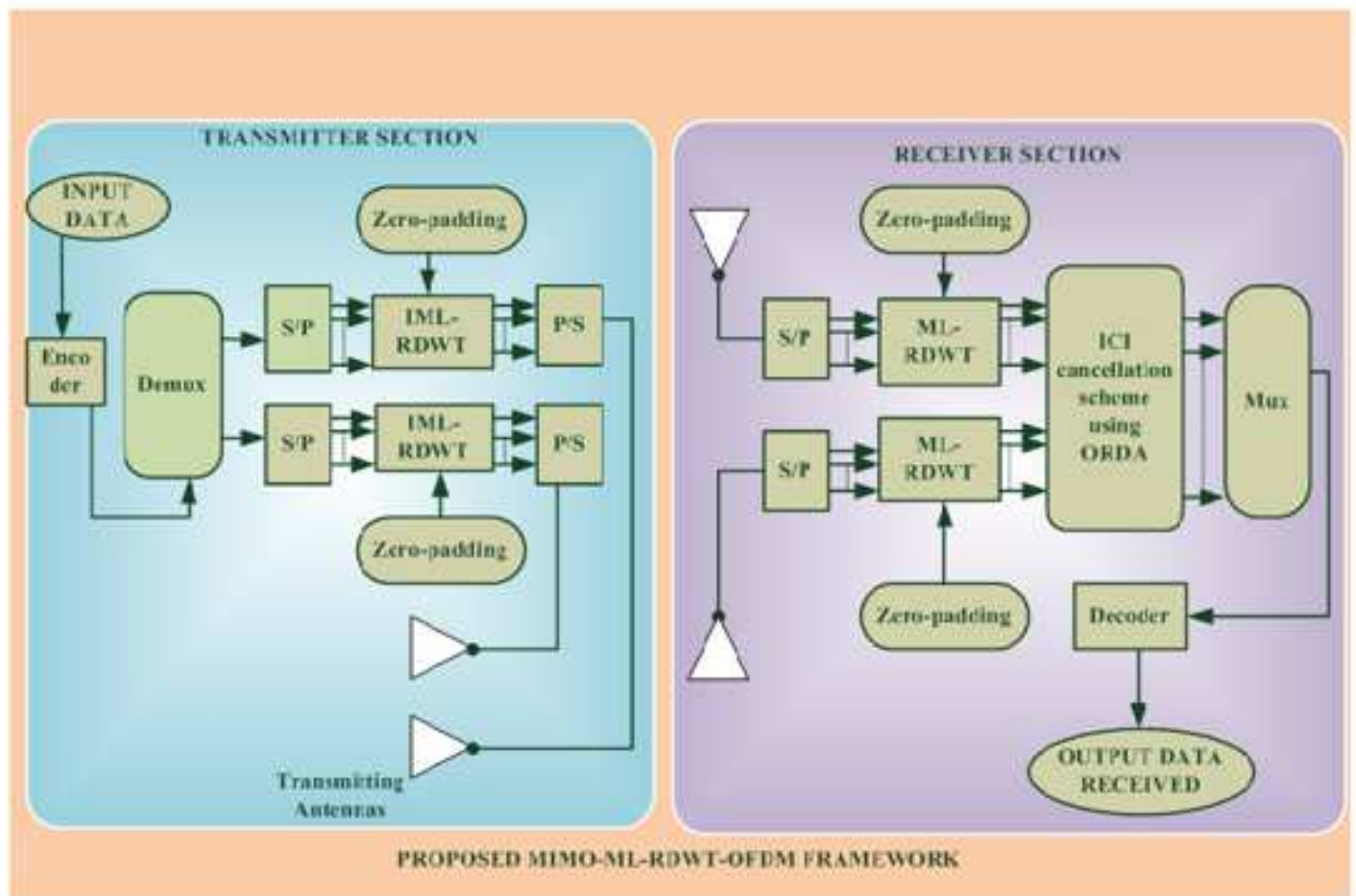

Figure 2

Architecture of proposed MIMO-ML-RDWT-OFDM system 


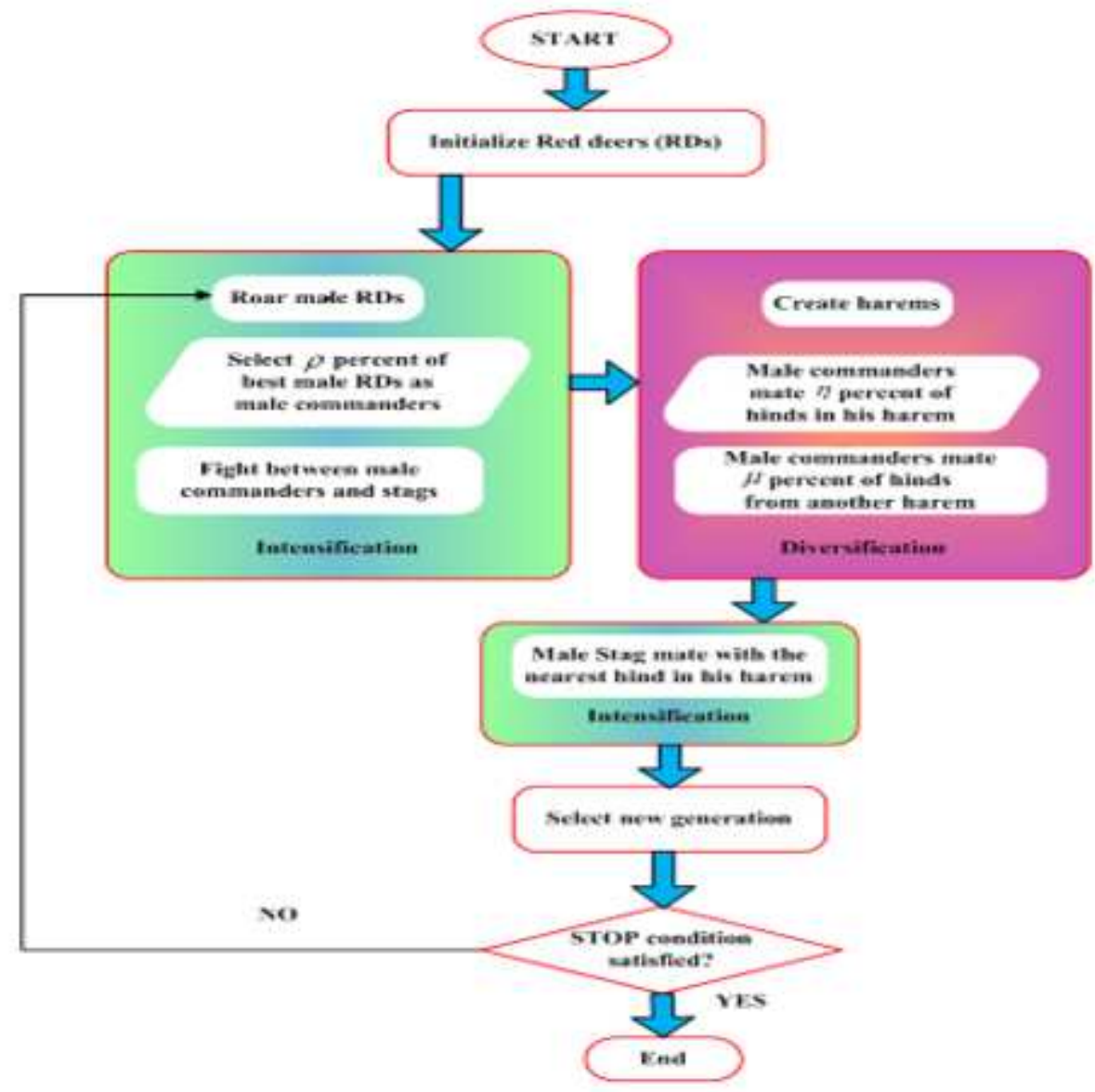

Figure 3

Flowchart model for ORDA

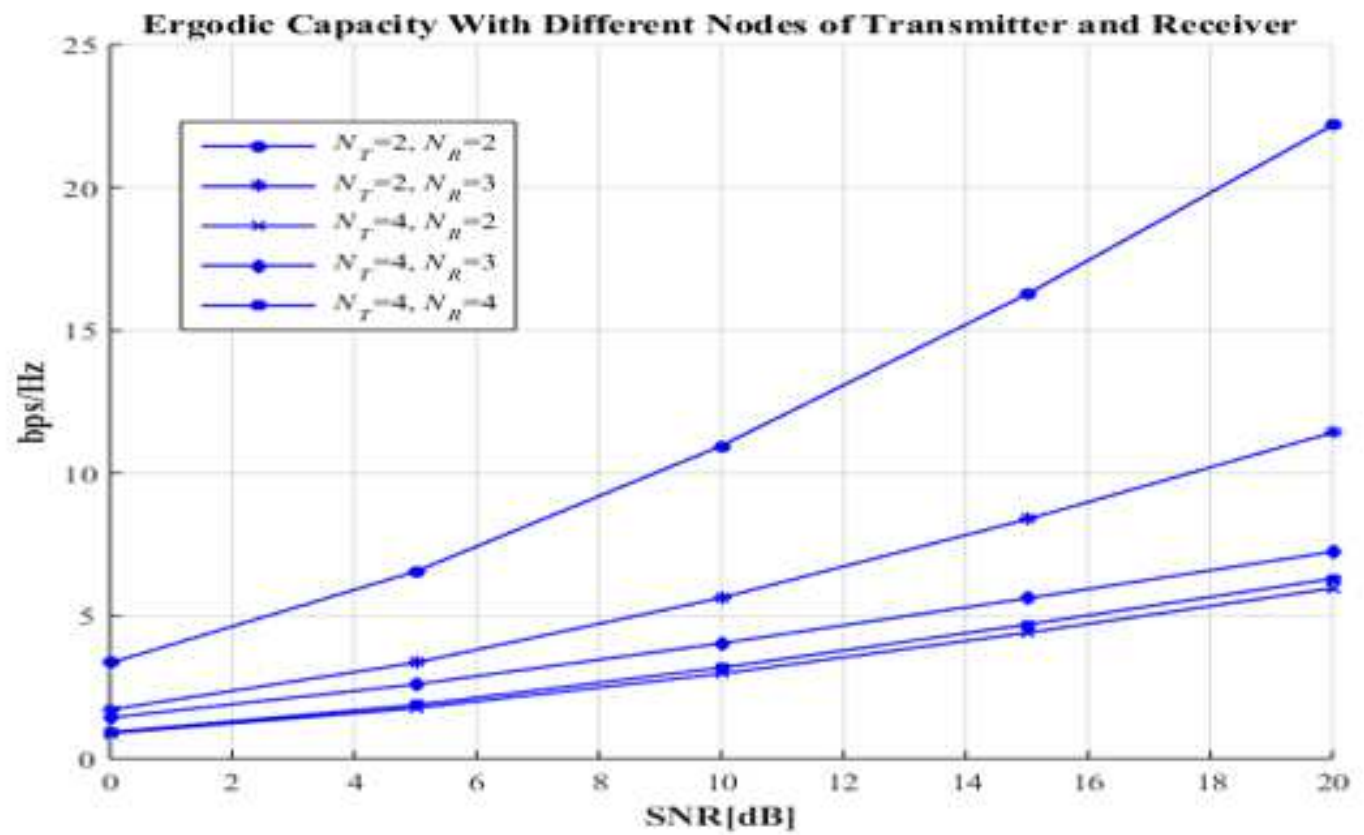

Figure 4 
Ergodic capacity for difference nodes of transmitter and receiver

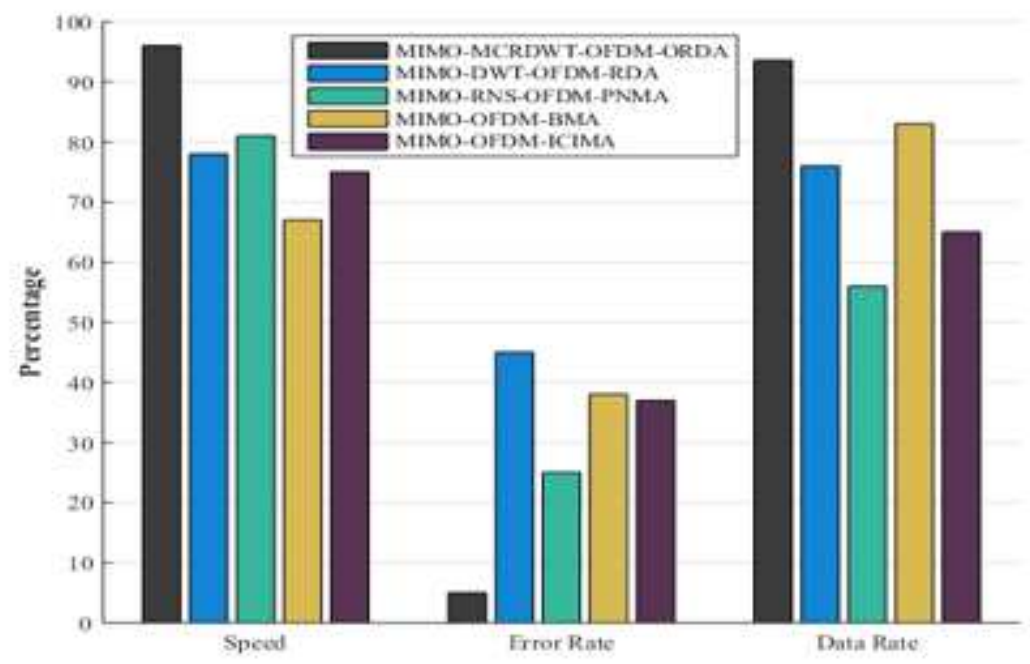

Figure 5

Comparison of speed, error rate and data rate of the proposed method with different existing methods

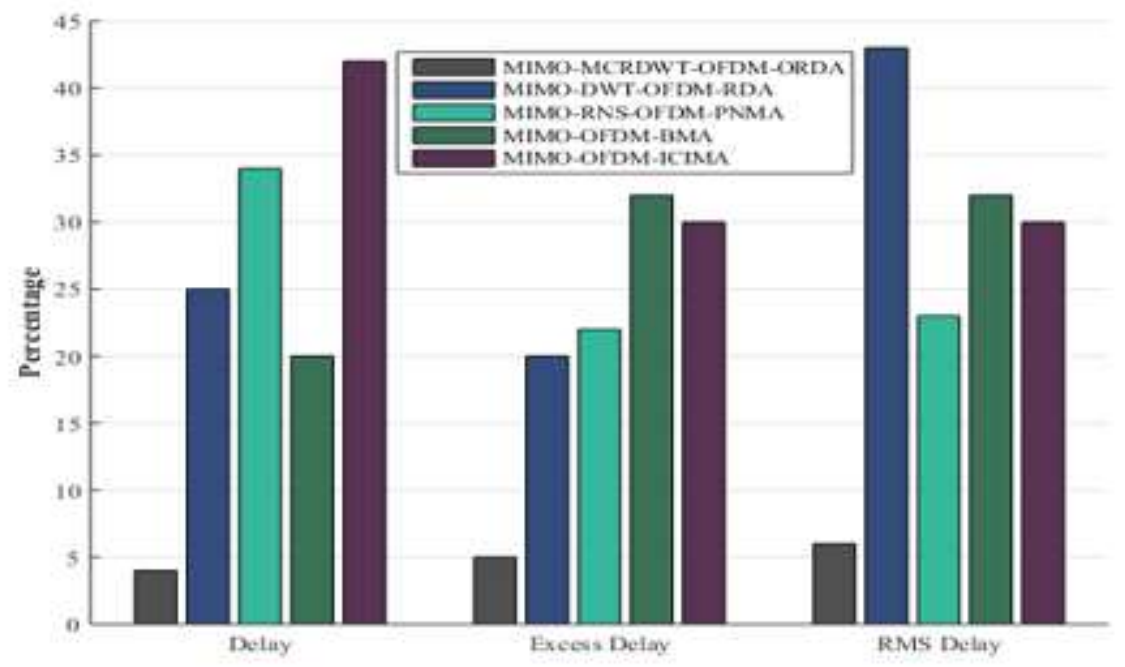

Figure 6

Comparison of various types of delay's in proposed method to that of existing methods 


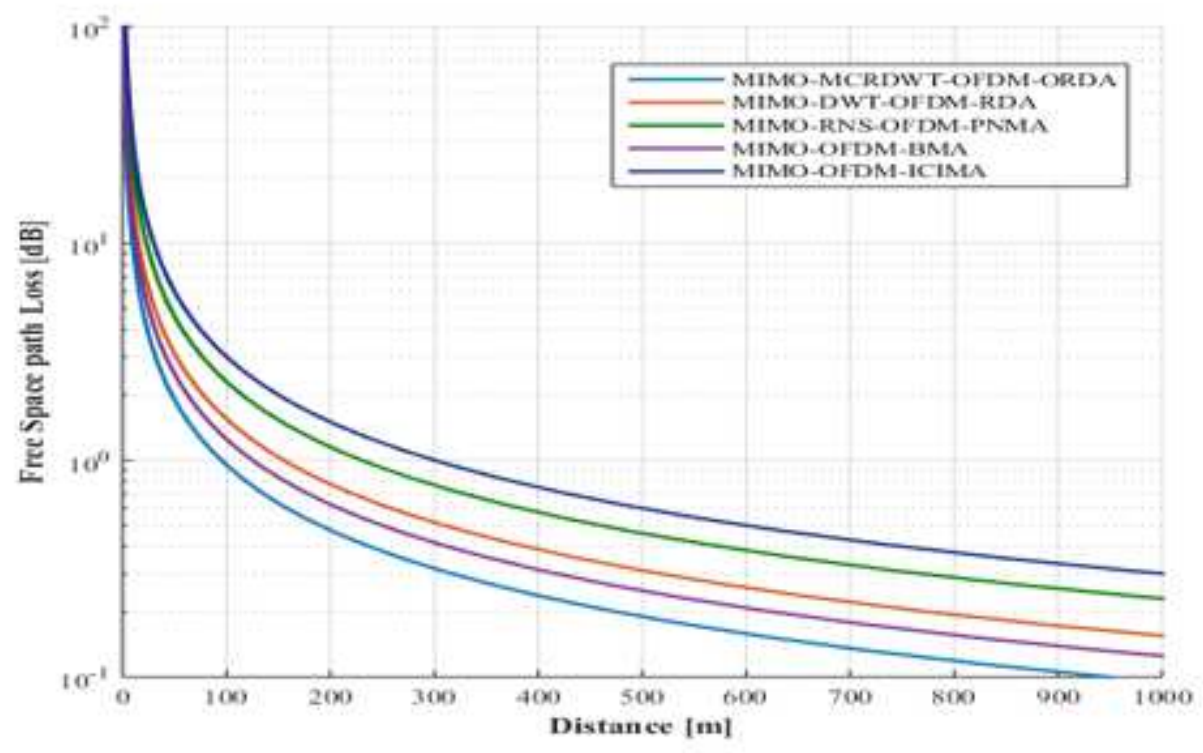

Figure 7

Comparison of Free space path loss for different distances

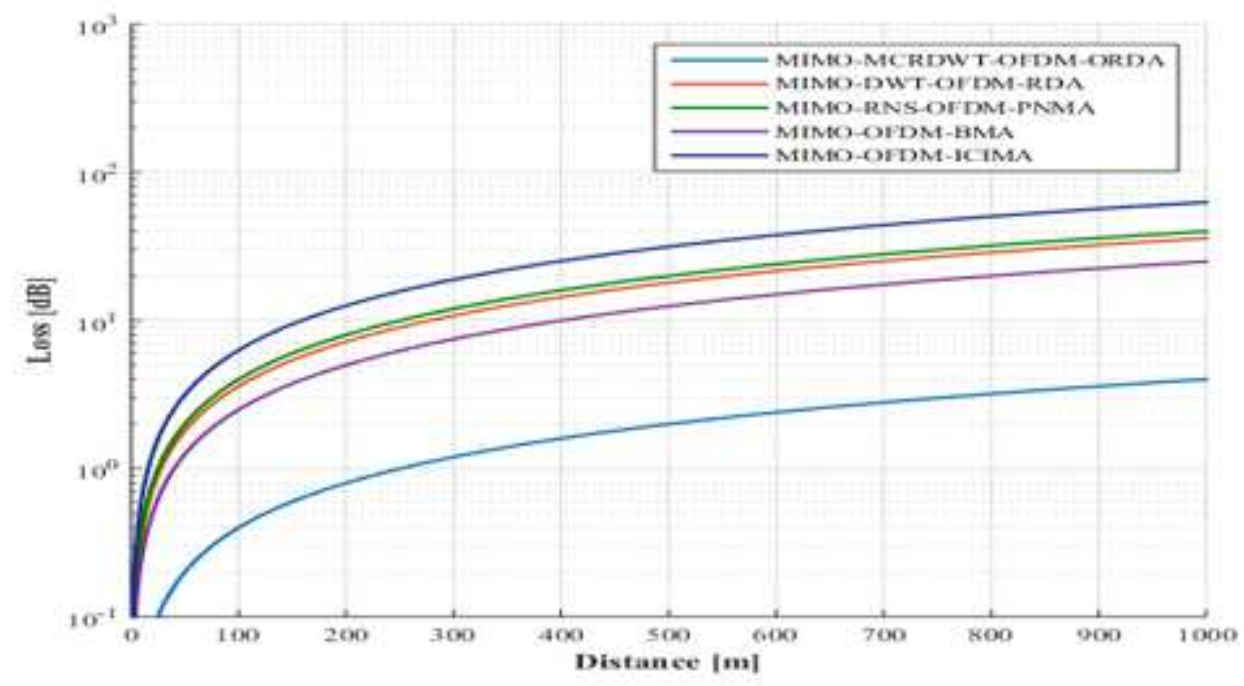

Figure 8

Comparison of loss for different distances 


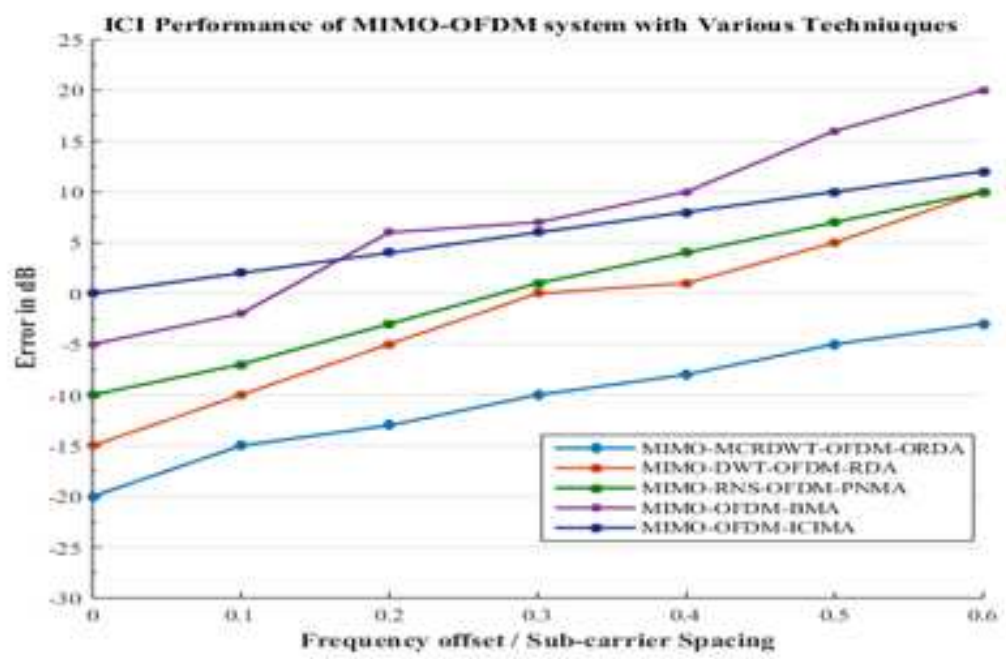

Figure 9

Comparison of error in proposed method and existing methods for different FO values

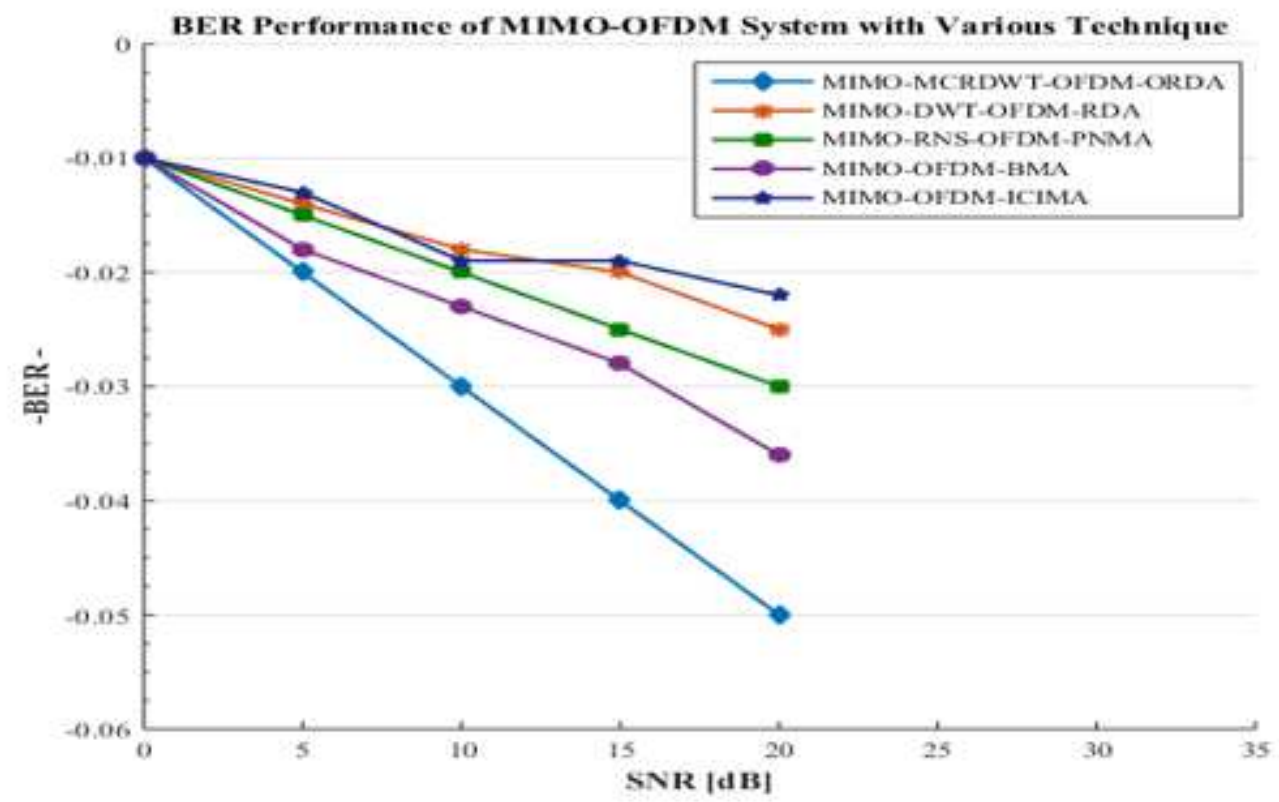

Figure 10

Comparison of BER and SNR in the proposed approach to that of the existing approaches. 


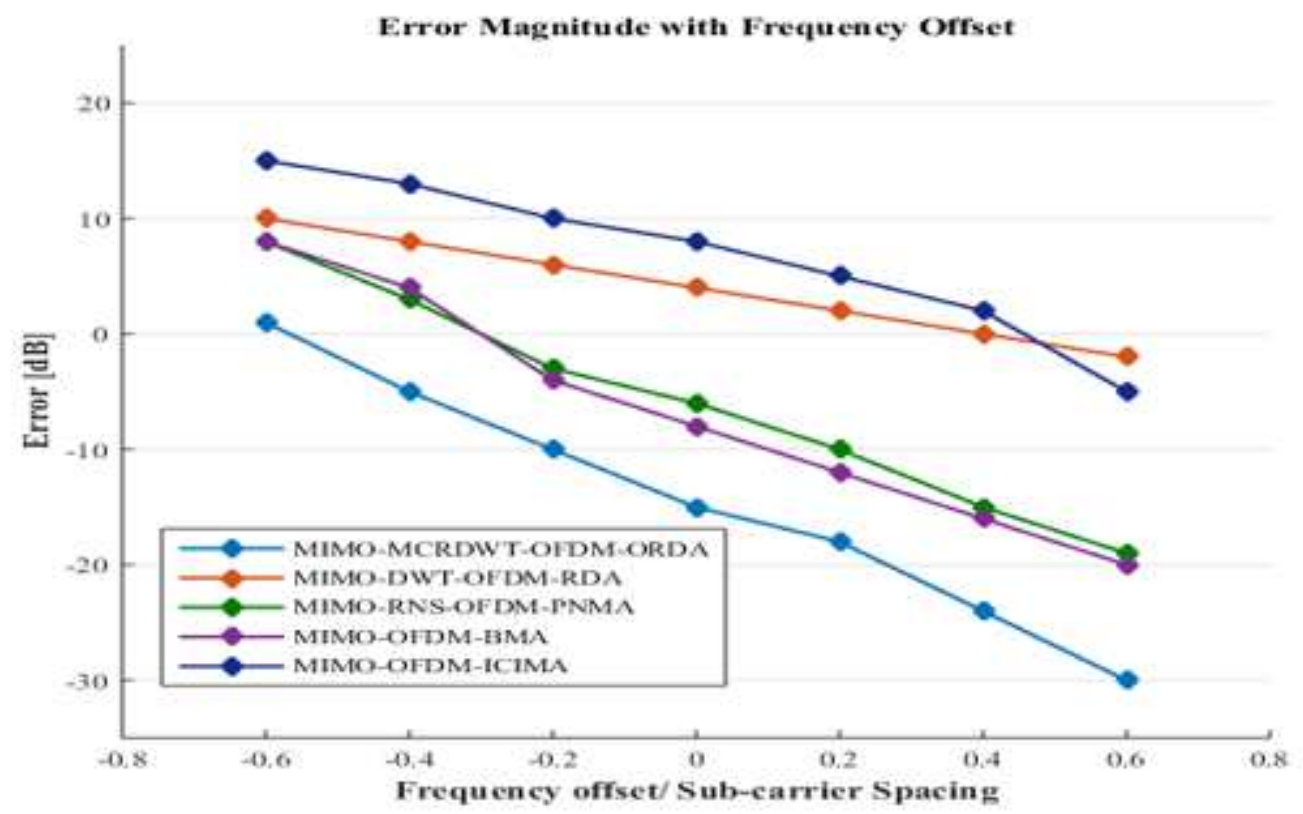

\section{Figure 11}

Comparison of error in the proposed method with various existing methods for different FO values.

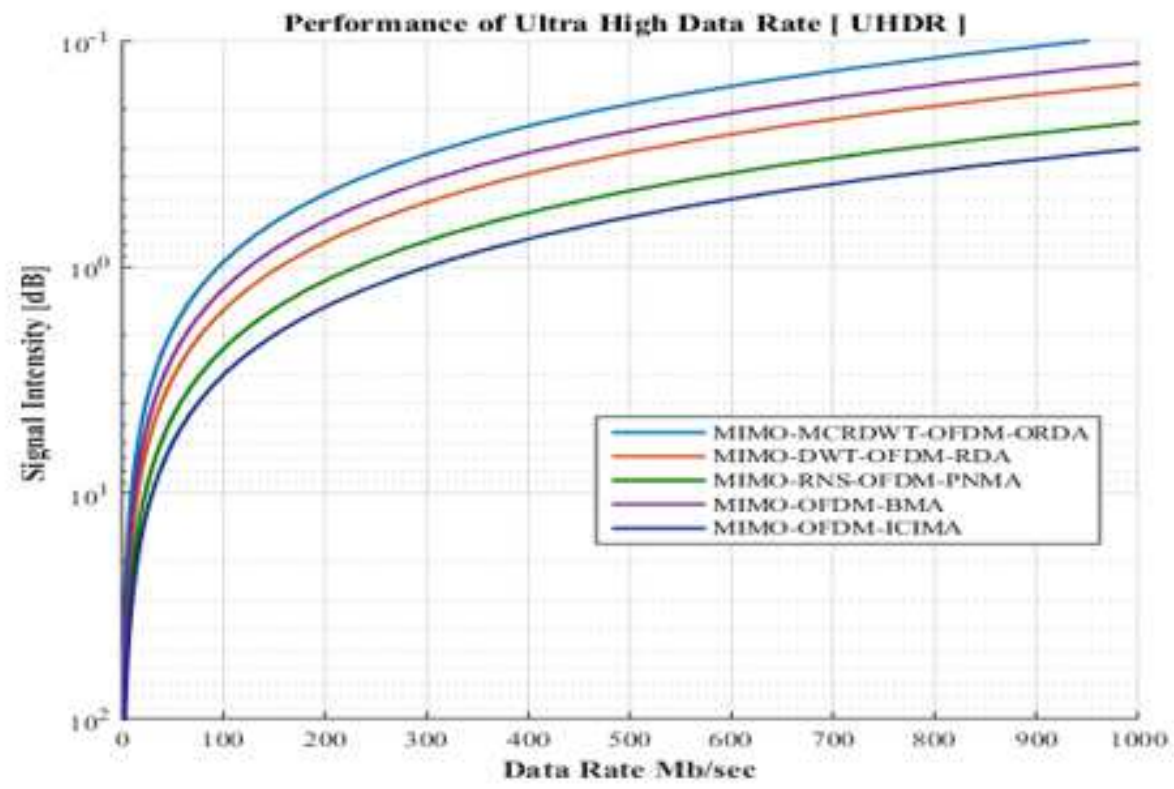

Figure 12

Comparison of signal intensity for varying data rates 


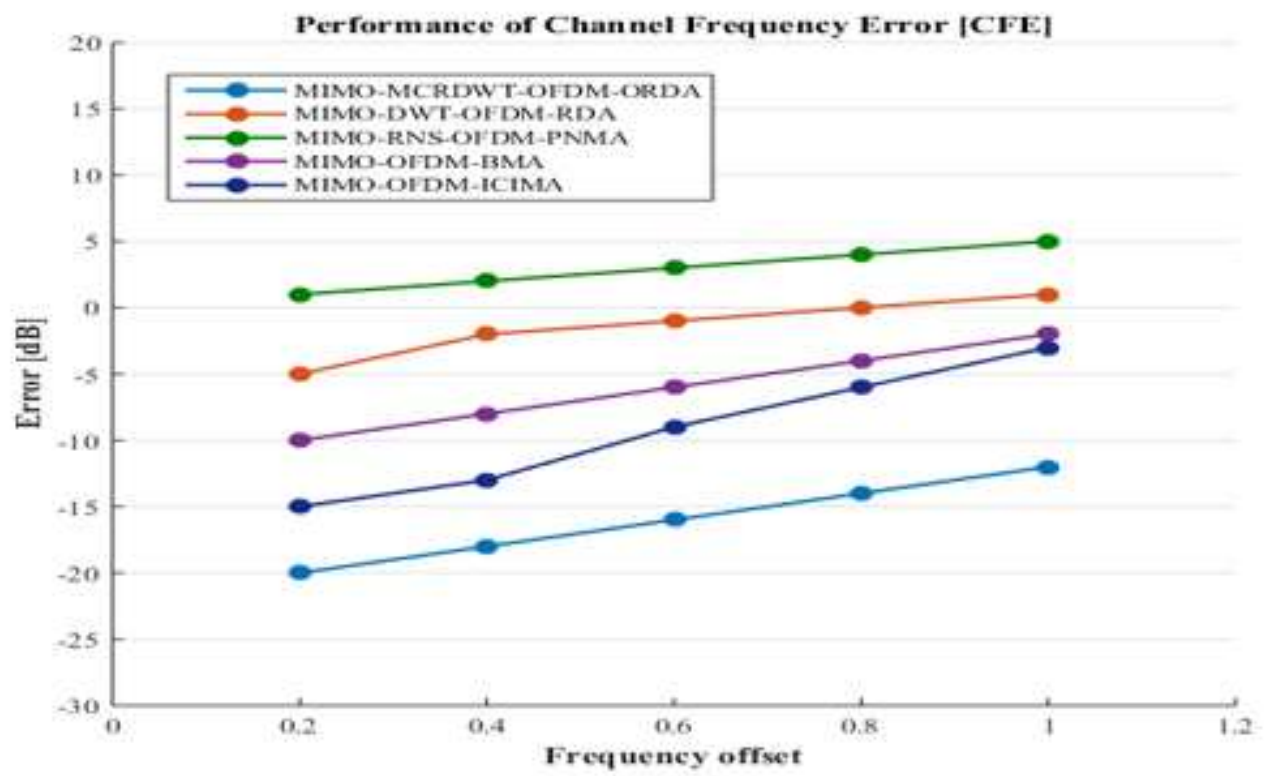

Figure 13

Comparison of channel frequency error for different FO values

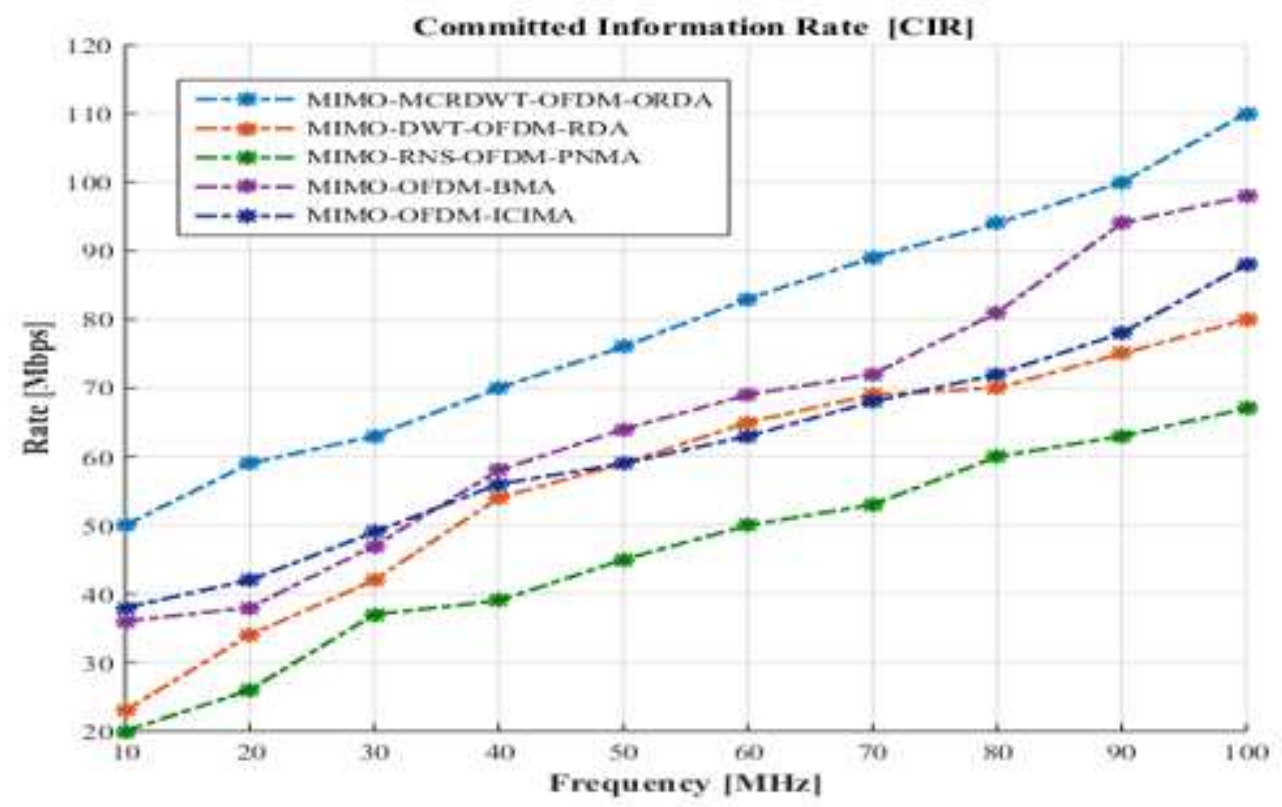

Figure 14

Comparison of Carrier-to-interference power ratio for different frequency values 


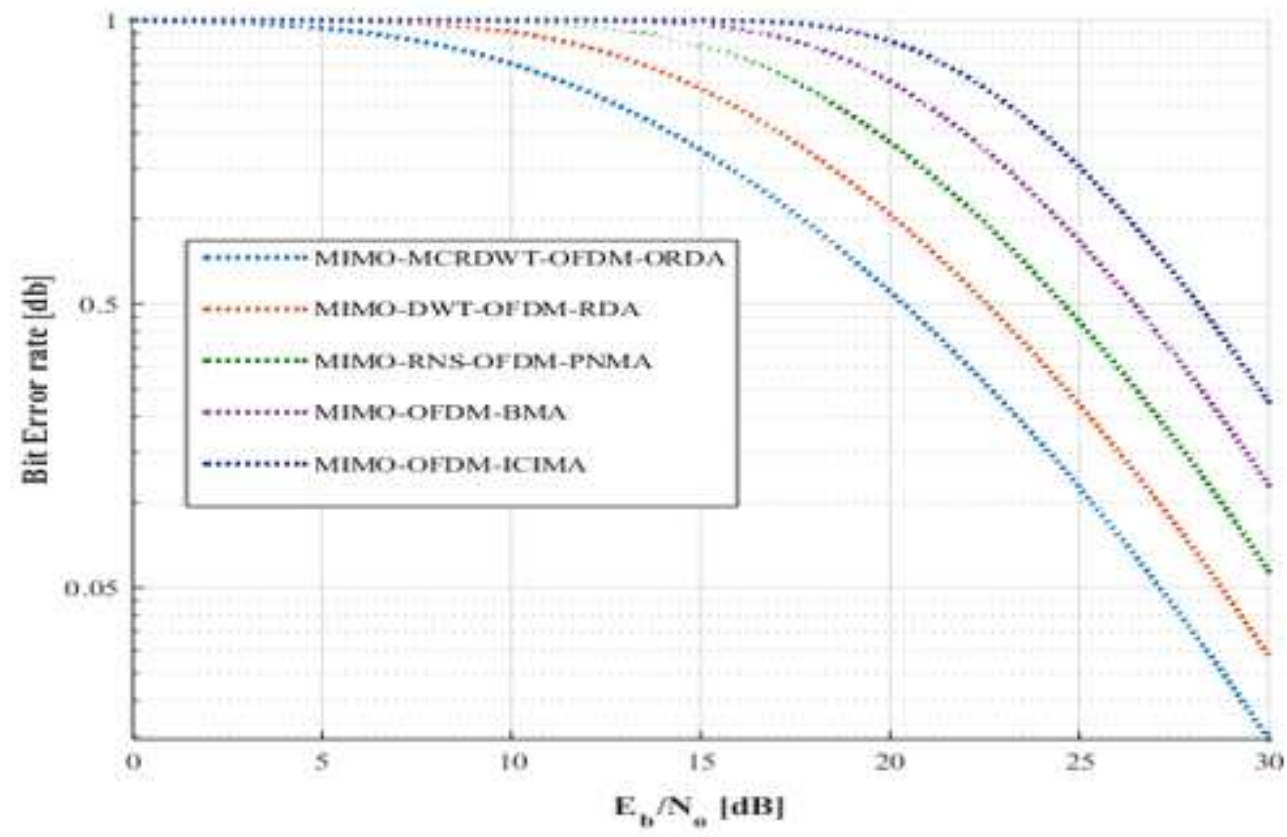

Figure 15

Comparisonof BER in the proposed approach to that of existing approaches

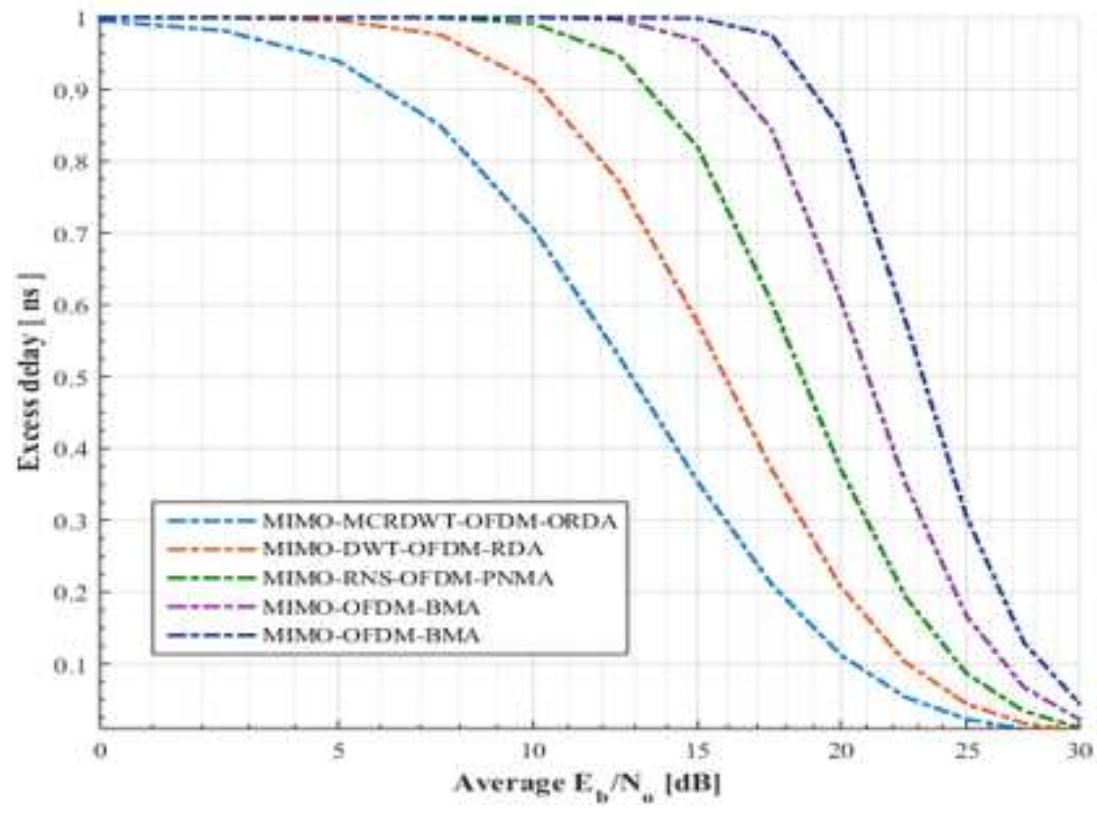

Figure 16

Comparison of excess delay in proposed method to that of the excess delay from various exiting methods 


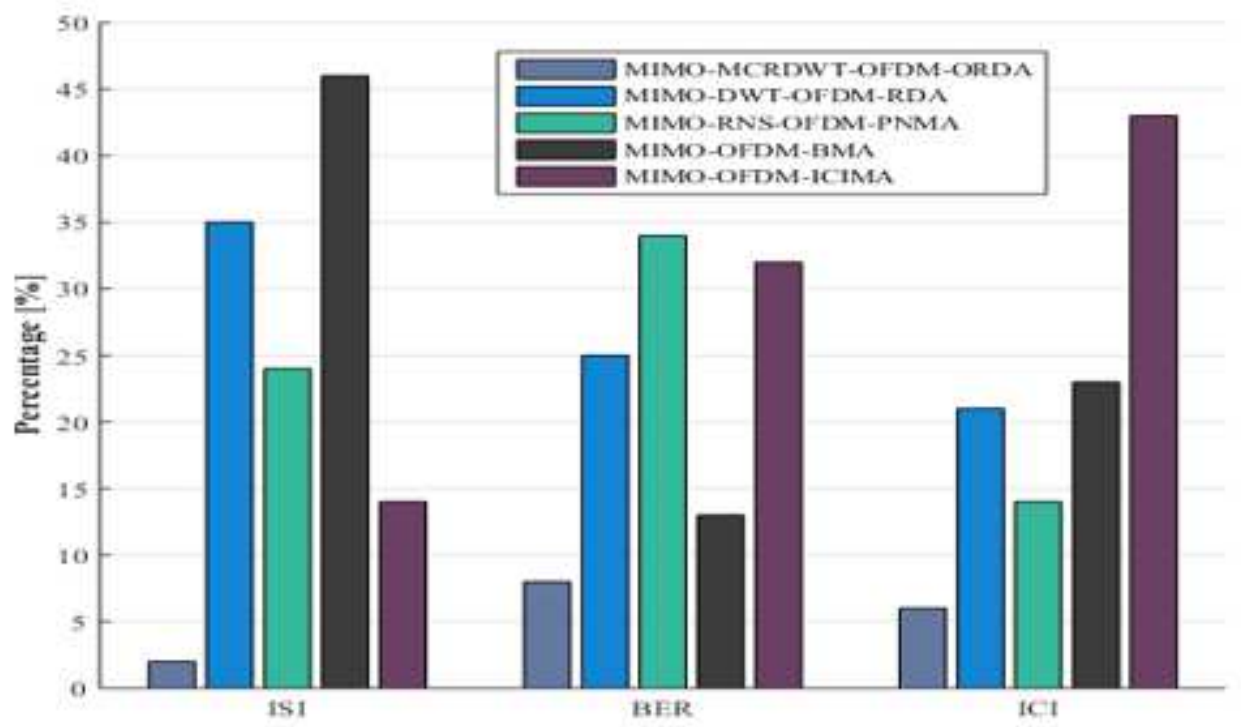

Figure 17

Comparison of ISI, BER and ICI in the proposed approach to that of the existing approaches 